\title{
Analysis of torus breakdown into chaos in a constraint Duffing van der Pol oscillator
}

\author{
Munehisa Sekikawa* \\ Aihara Complexity Modelling Project, ERATO, JST; \\ 3-23-5-2F Uehara, Shibuya-ku 151-0064, Japan \\ Institute of Industrial Science, The University of Tokyo; \\ 4-6-1 Komaba, Meguro-ku 153-8505, Japan \\ Naohiko Inaba \\ Inaba Research Laboratory; 1-45-1 Ekihigashi-dori, Oyama-shi 323-0022 Japan
}

Takashi Tsubouchi

Institute of Engineering Mechanics and Systems,

University of Tsukuba; 1-1-1 Ten'noudai, Tsukuba-shi, 305-8573, Japan

\author{
Kazuyuki Aihara \\ Institute of Industrial Science, The University of Tokyo; \\ 4-6-1 Komaba, Meguro-ku 153-8505, Japan \\ Aihara Complexity Modelling Project, ERATO, JST; \\ 3-23-5-2F Uehara, Shibuya-ku 151-0064, Japan
}




\begin{abstract}
The bifurcation structure of a constraint Duffing van der Pol oscillator with a diode is analyzed and an objective bifurcation diagram is illustrated in detail in this work. An idealized case, where the diode is assumed to operate as a switch, is considered.In this case, the Poincaré map is constructed as a one dimensional map: a circle map. The parameter boundary between a torusgenerating region where the circle map is a diffeomorphism and a chaos-generating region where the circle map has extrema is derived explicitly, without solving the implicit equations, by adopting some novel ideas. On the bifurcation diagram, intermittency and a saddle-node bifurcation from the periodic state to the quasi-periodic state can be exactly distinguished. Laboratory experiment is also carried out and theoretical results are verified.
\end{abstract}

keywords: chaos; torus; bifurcation; nonlinear oscillator

*Electronic address: muse@aihara.jst.go.jp 


\section{INTRODUCTION}

The purpose of this study is to analyze a simple driven oscillator in detail, and a bifurcation structure of its governing equation is discussed rigorously. The circuit that the present authors deal with in this paper is a constrain piecewise-linear Duffing van der Pol oscillator with a diode. Even though it is a natural and simple circuit, it possesses a rich variety of interesting bifurcation structures and phenomena such as the Arnol'd tongue, torus breakdown, and chaos. A parameter boundary between a torus-generating region and a chaos-generating region is explicitly derived and the objective detailed bifurcation is obtained.

Generally, even simple driven oscillators with only two dimensions have such complicated bifurcation structures that no comprehensive analysis of the structures covers all their aspects [Levi, 1981; Kawakami, 1984; Endo \& Chua, 1988; Mira \& Qriouet, 1993; Qriouet \& Mira, 1994; Qriouet \& Mira, 2000]. The conventional technique for the analysis of bifurcation structures of the driven oscillators is a mapping method, by which analysis of the continuous time system becomes the analysis of the discrete dynamics. However, it is difficult to analyze two- or higher dimensional discrete dynamics because mathematical discussions on such dynamics are too complex and their discussions are few. The literature indicate [Kawakami, 1984; Mira \& Qriouet, 1993; Qriouet \& Mira, 1994; Qriouet \& Mira, 2000] that it is an extremely difficult task to make the bifurcation structures clear completely even on the periodic states. There exist various locking states, namely infinitely many ultraharmonic, subharmonic and fractional harmonic entrainments which are well known as Arnol'd tongues. Also in these entrainments, saddle-node bifurcations, period-doubling bifurcations and NeimarkSacker bifurcations form an extremely complicated bifurcation structure [Mira \& Qriouet, 
1993; Qriouet \& Mira, 1994; Qriouet \& Mira, 2000].

On the other hand, bifurcation phenomena on one-dimensional maps have been investigated in detail [Collet \& Eckmann, 1980]. For example, the mechanism of period-doubling bifurcation is explained using the logistic map [Myrberg, 1963], and a definition of chaos on unimodal maps was given by $\mathrm{Li}$ and Yorke [Li \& Yorke, 1975]. The mechanism of various locking states and chaos via torus breakdown is explained using a circle map [Katznelson, 1977; Outlund et al., 1983; Kaneko, 1984].

Rössler first analyzed and introduced the constraint chaos generating differential equation to explain the qualitative aspects of chaos generation [Rössler, 1976; Rössler, 1979]. He showed that the chaos generation mechanism can be explained by the degenerate equation which consists of two 2-dimensional manifolds and one hysteresis. His smartness arises from the fact that the Hausdorff dimension [Young, 1983] of chaotic attractor generated in three dimensional autonomous oscillators is less than three. For such a system, the Poincaré map is almost one dimensional. Therefore, it is reasonable that Rössler attempted to explain generation of the chaos by means of one dimensional map.

There are some novel works about a forced relaxation oscillator where the Poincaré map is constructed as a one dimensional map: a circle map [Levi, 1981; Alseda \& Falco, 1994].

We aim at detailed analysis of a simpler driven circuit with a diode, and the objective two-parameter bifurcation diagram is illustrated in detail in this study. We introduce a piecewise-linear technique combined with a degeneration technique for this analysis. For a rigorous discussion on the bifurcation structure of this oscillator, we simplify the function of the diode to an ideal switch [Inaba \& Mori, 1991; Sekikawa et al., 2004]. If this idealization is simply applied, the order of the governing equation decreases to one lower order when the diode is on. From this degeneration, the Poincaré map is constructed as a circle map which 
is one-dimensional. The generation of interesting phenomena, such as the Arnol'd tongue, torus breakdown and chaos, is well explained by the Poincaré map.

We successfully derive the parameter boundary explicitly between a torus-generating region where the circle map is a diffeomorphism and a chaos-generating region where the circle map has extrema in a bifurcation diagram [Inaba \& Mori, 1991], which is denoted as $\Sigma$ hereafter. Generally, it is difficult to derive the boundary explicitly even if the Poincaré map is one-dimensional and a piecewise-linear technique is also applied. The reason is as follows: Although a solution of driven piecewise-linear oscillators can be obtained explicitly in each piecewise-linear branch, we must solve implicit equations to obtain the time when the solution crosses the boundary of the piecewise-linear branch. Therefore, the Poincaré map is given by implicit equations. This makes it difficult to analyze even simple piecewise-linear oscillators. In this study, we successfully obtained the bifurcation parameter $\Sigma$ explicitly without the need to solve the implicit equations, by adopting some novel ideas.

The main theorem for these ideas is presented in Section V. The objective bifurcation diagram of the oscillator is illustrated in detail. It must be mentioned that the bifurcation diagram was not obtained in [Inaba \& Mori 1991]. Arnol'd tongues and the parameter boundary $\Sigma$ are drawn in the diagram, where the saddle-node bifurcation from the periodic state to the quasi-periodic state is distinguished from intermittency. Moreover, laboratory experiment is also carried out and theoretical results are verified.

The novelty of this research work is that it provides physical substance for the extensively investigated analyses of the circle map. The degeneration of assuming the diode to be an ideal switch must be a simple and natural circuit model [Inaba \& Mori 1991; Sekikawa et al. 2004]. 



FIG. 1: Circuit diagram of Duffing van der Pol oscillator.

\section{CIRCUIT SETUP OF THE DUFFING VAN DER POL OSCILLATOR}

Figure 1 illustrates a circuit diagram with which the authors deal in this paper, where $L, C, R$, and $E \cos (\omega t)$ are an inductor, a capacitor, a linear resistor, and a forcing term, respectively. $-g_{N}$ is a linear negative conductance and $D$ is a diode. In this study, the $v-i$ characteristic of the diode is approximated as a piecewise-linear function:

$$
i_{d}(v)= \begin{cases}g_{d}(v-V) & \text { for } v \geq V \\ 0 & \text { for } v<V,\end{cases}
$$

where $g_{d}$ is assumed to be large. This is a natural assumption because the diode is a strongly nonlinear element. This characteristic is illustrated in Fig. 2. The governing equation is represented by the following 2nd-order nonautonomous differential equation:

$$
\left\{\begin{array}{l}
C \frac{d v}{d t}=g_{N} v-i_{d}(v)+i \\
L \frac{d i}{d t}=-v-R i+E \cos (\omega t) .
\end{array}\right.
$$




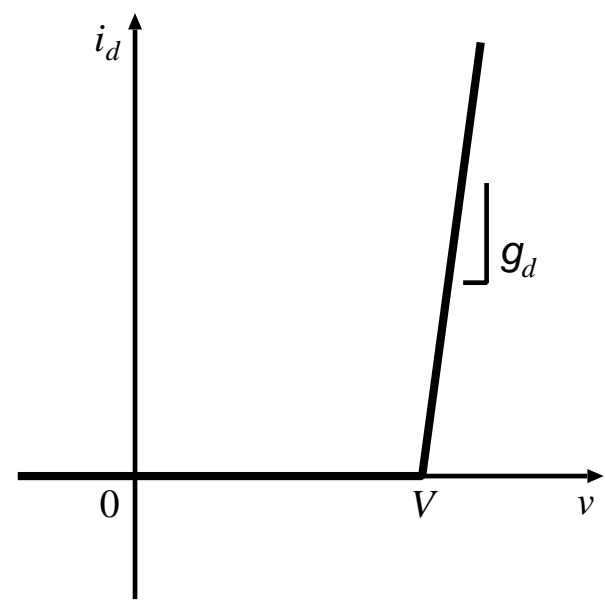

FIG. 2: $v-i$ characteristic of diode.

If diode $D$ is replaced with a diode pair, and the $v-i$ characteristics of the pair is approximated by a third-order polynomial function, then the governing equation is represented by the Duffing van der Pol equation [Ueda \& Akamatsu, 1981]. The "Duffing van der Pol" qualifier implies the presence of a nonlinear restoring term, and a nonlinear damping with negative and positive components in the equation. As the circuit in this paper contains only a single diode, the nonlinearity $i_{d}(v)$ in Eq. (2) becomes asymmetric, which is represented by a piecewise-linear function. Therefore, this equation can be recognized as a piecewise-linear version of the Duffing van der Pol equation with asymmetric nonlinearity.

Changing the variables and parameters in Eqs. (1) and (2) as

$$
\begin{gathered}
x=\frac{1}{V} v, \quad y=\frac{1}{V} \sqrt{\frac{L}{C}} i, \quad \tau=\frac{1}{\sqrt{L C}} t, \\
\delta_{1}=\frac{R}{2} \sqrt{\frac{C}{L}}, \quad \delta_{2}=\frac{g_{N}}{2} \sqrt{\frac{L}{C}}, \quad \alpha=\frac{g_{d}}{2} \sqrt{\frac{L}{C}} \\
\nu=\sqrt{L C} \omega, \quad B=\frac{E}{V},
\end{gathered}
$$


yields the normalized equation, which is represented by

$$
\begin{aligned}
& \left\{\begin{array}{l}
\dot{x}=2 \delta_{2} x-h(x)+y, \\
\dot{y}=-x-2 \delta_{1} y+B \cos (\nu \tau),
\end{array}\right. \\
& h(x)= \begin{cases}2 \alpha(x-1) & \text { for } x \geq 1 \\
0 & \text { for } x<1,\end{cases}
\end{aligned}
$$

where ' $'$ means a derivation of $\tau$ and $\alpha \gg 1$ is assumed. This assumption corresponds to the fact that $g_{d}$ is assumed to be large, which is closely related to the constraint equation proposed in the next section.

The characteristic equations of Eq. (4) are given by

$$
\begin{array}{ll}
\lambda^{2}+2\left(\delta_{1}-\delta_{2}+\alpha\right) \lambda+\left(1-4 \delta_{1}\left(\delta_{2}-\alpha\right)\right)=0 & \text { for } x \geq 1, \quad(a) \\
\lambda^{2}+2\left(\delta_{1}-\delta_{2}\right) \lambda+\left(1-4 \delta_{1} \delta_{2}\right)=0 & \text { for } x<1 .
\end{array}
$$

The eigenvalues of Eq. (5.a) in the region where $x \geq 1$ are assumed to be real and denoted by $\sigma_{1}$ and $\sigma_{2}$, where $\left|\sigma_{1}\right|>\left|\sigma_{2}\right|$ is assumed. Those of Eq. (5.b) in the region where $x<1$ are complex and denoted by $\delta \pm j \gamma$. Since Eq. (4) is piecewise-linear, the explicit solution is obtained in each branch. Let $\left(x\left(\tau ; \tau_{0}, x_{0}, y_{0}\right), y\left(\tau ; \tau_{0}, x_{0}, y_{0}\right)\right)^{T}$ be the flow of Eq. (4) for the initial condition given by $(\tau, x, y)=\left(\tau_{0}, x_{0}, y_{0}\right)$. The explicit solution is represented by the 
following equations. If $x \geq 1$, then

$$
\begin{aligned}
& \left(\begin{array}{l}
x\left(\tau ; \tau_{0}, x_{0}, y_{0}\right) \\
y\left(\tau ; \tau_{0}, x_{0}, y_{0}\right)
\end{array}\right)=F_{1}\left(\tau-\tau_{0}\right) \times\left(\begin{array}{c}
C_{1} \\
C_{2}
\end{array}\right)+G_{1}(\tau) \\
& \text { where } \quad F_{1}(\tau)=\left(\begin{array}{c}
f_{1 a}(\tau) \\
f_{1 b}(\tau)
\end{array}\right), G_{1}(\tau)=\left(\begin{array}{c}
g_{1 a}(\tau) \\
g_{1 b}(\tau)
\end{array}\right) \\
& f_{1 a}(\tau)=\left(e^{\sigma_{1} \tau} \quad e^{\sigma_{2} \tau}\right), \quad g_{1 a}(\tau)=k_{1 a} \sin (\nu \tau)+k_{1 b} \cos (\nu \tau)+k_{1 c} \\
& f_{1 b}(\tau)=\frac{d}{d \tau} f_{1 a}(\tau)-2\left(\delta_{2}-\alpha\right) f_{1 a}(\tau) \\
& g_{1 b}(\tau)=\frac{d}{d \tau} g_{1 a}(\tau)-2\left(\delta_{2}-\alpha\right) g_{1 a}(\tau)-2 \alpha \\
& k_{1 a}=\frac{-2\left(\delta_{2}-\alpha-\delta_{1}\right) \nu B}{\left(1-4 \delta_{1}\left(\delta_{2}-\alpha\right)-\nu^{2}\right)^{2}+4\left(\delta_{2}-\alpha-\delta_{1}\right)^{2} \nu^{2}} \\
& k_{1 b}=\frac{\left(1-4 \delta_{1}\left(\delta_{2}-\alpha\right)-\nu^{2}\right) B}{\left(1-4 \delta_{1}\left(\delta_{2}-\alpha\right)-\nu^{2}\right)^{2}+4\left(\delta_{2}-\alpha-\delta_{1}\right)^{2} \nu^{2}} \\
& k_{1 c}=\frac{4 \delta_{1} \alpha}{1-4 \delta_{1}\left(\delta_{2}-\alpha\right)},
\end{aligned}
$$

and $C_{1}$ and $C_{2}$ are constants that satisfy

$$
\left(\begin{array}{c}
C_{1} \\
C_{2}
\end{array}\right)=F_{1}(0)^{-1} \times\left\{\left(\begin{array}{l}
x_{0} \\
y_{0}
\end{array}\right)-G_{1}\left(\tau_{0}\right)\right\} .
$$

If $x<1$, then

$$
\begin{aligned}
& \left(\begin{array}{c}
x\left(\tau ; \tau_{0}, x_{0}, y_{0}\right) \\
y\left(\tau ; \tau_{0}, x_{0}, y_{0}\right)
\end{array}\right)=F_{2}\left(\tau-\tau_{0}\right) \times\left(\begin{array}{l}
C_{3} \\
C_{4}
\end{array}\right)+G_{2}(\tau) \\
& \text { where } \quad F_{2}(\tau)=\left(\begin{array}{c}
f_{2 a}(\tau) \\
f_{2 b}(\tau)
\end{array}\right), G_{2}(\tau)=\left(\begin{array}{c}
g_{2 a}(\tau) \\
g_{2 b}(\tau)
\end{array}\right) \\
& f_{2 a}(\tau)=\left(e^{\delta \tau} \sin (\gamma \tau) e^{\delta \tau} \cos (\gamma \tau)\right), \quad g_{2 a}(\tau)=k_{2 a} \sin (\nu \tau)+k_{2 b} \cos (\nu \tau) \\
& f_{2 b}(\tau)=\frac{d}{d \tau} f_{2 a}(\tau)-2 \delta_{2} f_{2 a}(\tau), g_{2 b}(\tau)=\frac{d}{d \tau} g_{2 a}(\tau)-2 \delta_{2} g_{2 a}(\tau), \\
& k_{2 a}=\frac{-2\left(\delta_{2}-\delta_{1}\right) \nu B}{\left(1-4 \delta_{1} \delta_{2}-\nu^{2}\right)^{2}+4\left(\delta_{2}-\delta_{1}\right)^{2} \nu^{2}}, \quad k_{2 b}=\frac{\left(1-4 \delta_{1} \delta_{2}-\nu^{2}\right) B}{\left(1-4 \delta_{1} \delta_{2}-\nu^{2}\right)^{2}+4\left(\delta_{2}-\delta_{1}\right)^{2} \nu^{2}} \\
& \delta=-\left(\delta_{1}-\delta_{2}\right), \gamma=\sqrt{1-\left(\delta_{1}+\delta_{2}\right)^{2}},
\end{aligned}
$$


and $C_{3}$ and $C_{4}$ are constants that satisfy

$$
\left(\begin{array}{c}
C_{3} \\
C_{4}
\end{array}\right)=F_{2}(0)^{-1} \times\left\{\left(\begin{array}{l}
x_{0} \\
y_{0}
\end{array}\right)-G_{2}\left(\tau_{0}\right)\right\} .
$$

To draw the attractors of Eq. (4), the solutions, Eqs. (6) and (8), must be alternated and connected continuously at the boundary of $x=1$. At this boundary $(x=1)$, the values of $y$ and $\tau$ must be obtained for the continuous connection, that is, these values are necessary for the initial conditions in order to draw the solution after the flow passes over the boundary. To derive time $\tau$, we must solve the transcendental equation $x(\tau)=1$. This equation is solved by Newton's method. After this $\tau$ is obtained, substituting $\tau$ into Eq. (6) or (8) yields $y$.

Figures 3 and 4 illustrate the attractors observed experimentally and numerically, respectively. The amplitude of the forcing term is chosen as a varying parameter. As shown in these figures, for smaller amplitude, a quasi-periodic attractor is observed (Figs. 3 (a) and $4(\mathrm{a})$ ), and for larger amplitude, a chaotic attractor is observed (Figs. 3 (c) and 4 (c)). Periodic attractors are observed at several amplitudes (Figs. 3 (b) and 4 (b)).

\section{CIRCUIT SETUP WITH IDEALIZED DIODE}

Because of the strong nonlinearity of the diode, $\alpha \gg 1$ was naturally assumed in the last section. If we take a limit of $\alpha \rightarrow \infty$ in Eq. (4), then the diode can be recognized as an ideal switch. The $v-i$ characteristic of the idealized diode is illustrated in Fig. 5. We study Eq. (4) under this simplified function of the diode. At the end of this section, we will demonstrate that attractors based on this rigorous study with the simplified diode (Fig. 6) are identical with those in Fig. 4 in Section II.

The governing equation under the assumption of the idealized diode is represented by the 


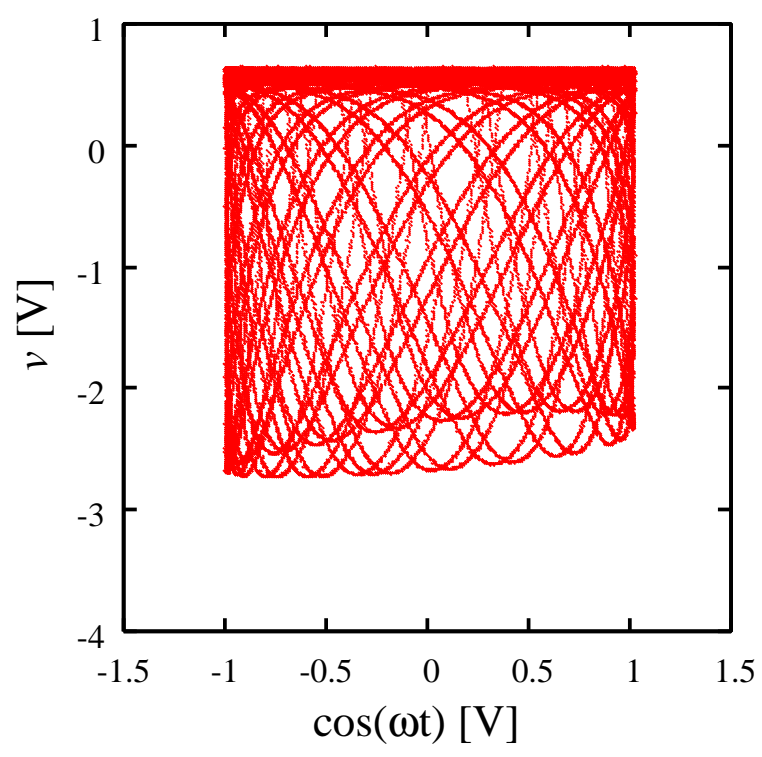

(a)

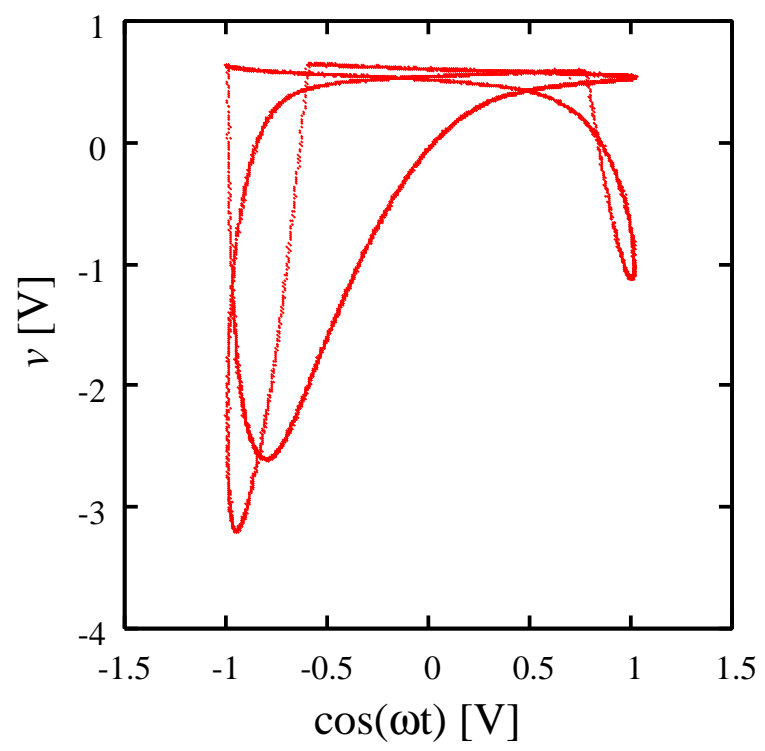

(b)

FIG. 3: Attractors observed in laboratory experiment with $L=47 \mathrm{mH}, C=0.01 \mu \mathrm{F}$, $R=140 \Omega, g_{N}=4.7 \mathrm{mS}$, (The natural frequency is $3.8 \mathrm{kHz}$.) and $\omega=2.7 \mathrm{kHz}$ :

(a) Quasi-periodic attractor with $E=0.05 \mathrm{~V}$; (b) Periodic attractor with $E=0.24 \mathrm{~V}$;

(c) Chaotic attractor with $E=0.32 \mathrm{~V}$. 


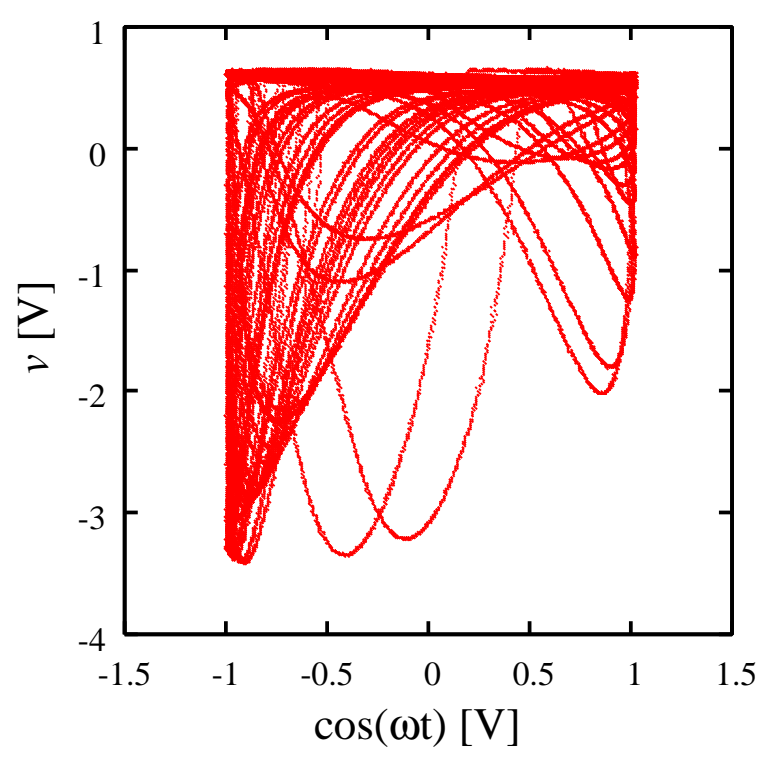

(c)

FIG. 3: - continued.

following constraint equations [Inaba et al., 1987; Inaba \& Mori, 1991; Sekikawaet al.]:

$$
\begin{aligned}
& 1 \text { diode on }:\left\{\begin{array}{l}
x=1(\text { constant }), \\
\dot{y}=-2 \delta_{1} y-1+B \cos (\nu \tau),
\end{array}\right. \\
& 2 \text { diode off : }\left\{\begin{array}{l}
\dot{x}=2 \delta_{2} x+y, \\
\dot{y}=-x-2 \delta_{1} y+B \cos (\nu \tau) .
\end{array}\right.
\end{aligned}
$$

When the diode is on, $x=1$ (constant), because the voltage across the capacitor $v$ is constrained to the threshold voltage $V$ of the diode. In turn, when the diode is off, Eq. (10.b) is the same as Eq. (4). It must be noted that the governing equation for the region in which diode is on is of the first order because $x$ is constant. Solutions of Eq. (10) are connected by the following transition conditions:

$$
\left\{\begin{array}{l}
\mathbf{1} \longrightarrow \mathbf{2}: y=-2 \delta_{2},(a) \\
\mathbf{2} \longrightarrow \mathbf{1}: x=1
\end{array}\right.
$$




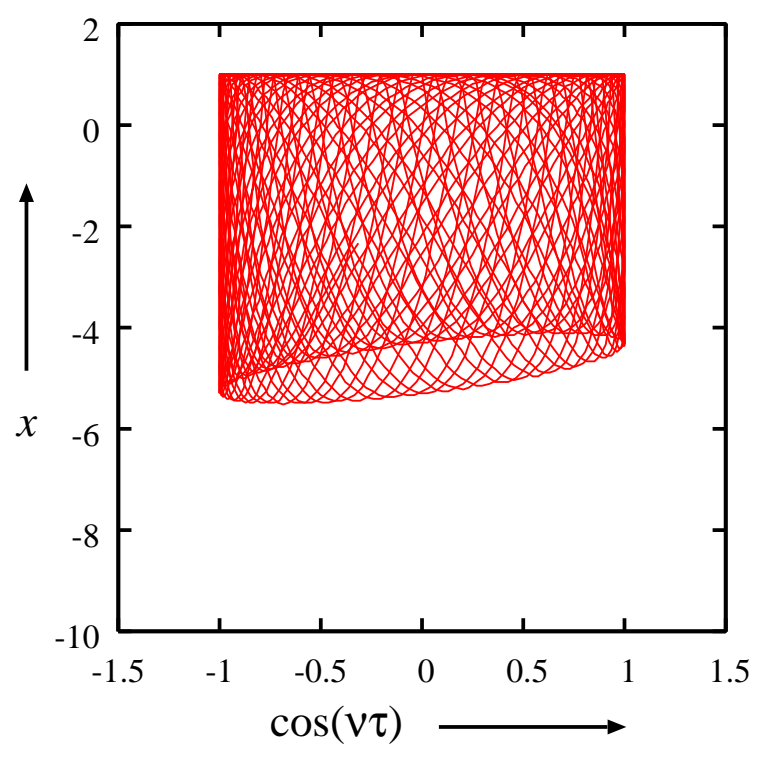

(a)

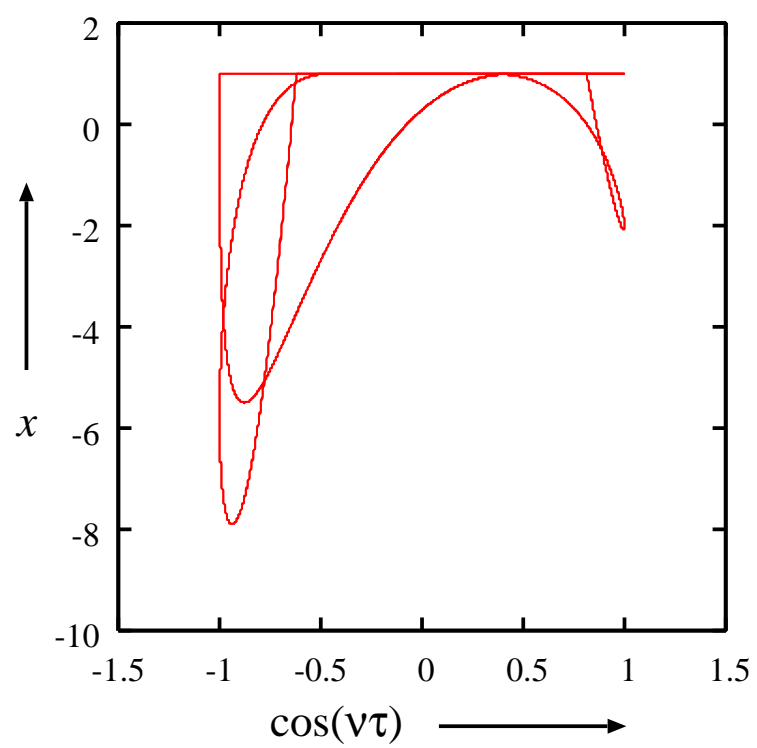

(b)

FIG. 4: Attractors obtained from Eq. (4) with $\delta_{1}=0.1, \delta_{2}=0.5, \alpha=1000$, and $\nu=0.745$ :

(a) Quasi-periodic attractor with $B=0.1$; (b) Periodic attractor with $B=0.3$;

(c) Chaotic attractor with $B=0.38$. 


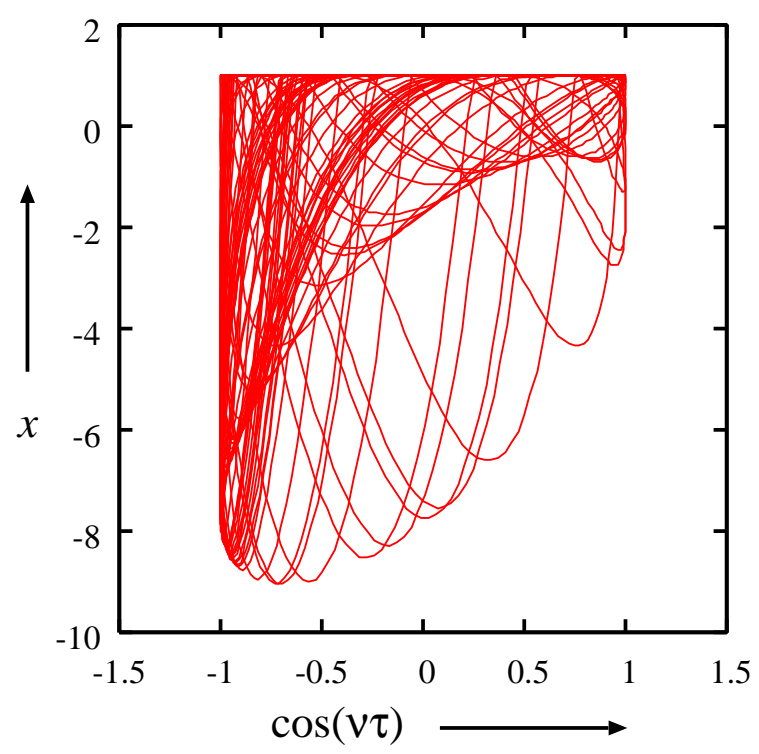

(c)

FIG. 4: - continued.

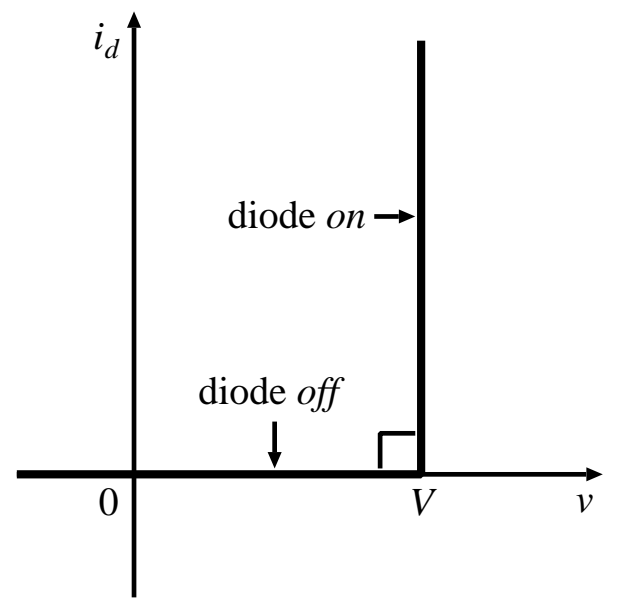

FIG. 5: $v-i$ characteristic of the idealized diode.

Equation (10.a) takes the governing equation from Eq. (10.b) when the voltage across the diode reaches the threshold voltage corresponding to Eq. (11.b). In turn, Eq. (10.b) takes the governing equation from Eq. (10.a) when the current through the diode decreases to zero, corresponding to Eq. (11.a). This degeneration technique is proposed by the authors 
in order to analyze third-order autonomous circuits [Inaba et al., 1987; Inaba \& Mori, 1988] or second-order nonautonomous circuits [Inaba \& Mori, 1991; Sekikawa et al.] with chaosgenerating capability. The governing equation of the circuit in the diode off region $(x<1)$ is represented by Eq. (4), and therefore, the solution is given by Eq. (8). On the other hand, the solution of the diode on region $(x=1)$ is given by

1 diode on :

$$
\begin{aligned}
& y\left(\tau ; \tau_{0}, y_{0}\right)=C_{5} e^{-2 \delta_{1}\left(\tau-\tau_{0}\right)}+k_{1 a} \sin (\nu \tau)+k_{1 b} \cos (\nu \tau)+k_{1 c} \\
& \text { where } \quad k_{1 a}=\frac{\nu B}{\nu^{2}+4 \delta_{1}^{2}}, k_{1 b}=\frac{2 \delta_{1} B}{\nu^{2}+4 \delta_{1}^{2}}, k_{1 c}=-\frac{1}{2 \delta_{1}}
\end{aligned}
$$

and $C_{5}$ is a constant that satisfies

$$
C_{5}=y_{0}-k_{1 a} \sin \left(\nu \tau_{0}\right)-k_{1 b} \cos \left(\nu \tau_{0}\right)-k_{1 c} .
$$

Figure 6 shows the attractors obtained from the constraint equations (10) and (11). The maximum Lyapunov exponent is also written in the caption. The derivation of the maximum Lyapunov exponent is described later. Figure 6 (a) shows a quasi-periodic attractor, and Figs. 6 (b) and (c) show a periodic and a chaotic attractor, respectively.

Equations (10) and (11) are a good representative of Eq. (4) if $\alpha \gg 1$, because Eqs. (10) and (11) under $\alpha \gg 1$ yield various phenomena observed from Eq. (4), as shown in Figs. 4 and 6 . In the next section, we will analyze these interesting phenomena rigorously using these constraint Eqs. (10) and (11).

\section{CONSTRUCTION OF POINCARÉ MAP}

The conventional analysis of the bifurcation structure of driven oscillators is a mapping method [Kawakami, 1984] by which the analysis of differential equations of the continuous time system arrives at that of the discrete dynamics. However, it is difficult to analyze 




(a)

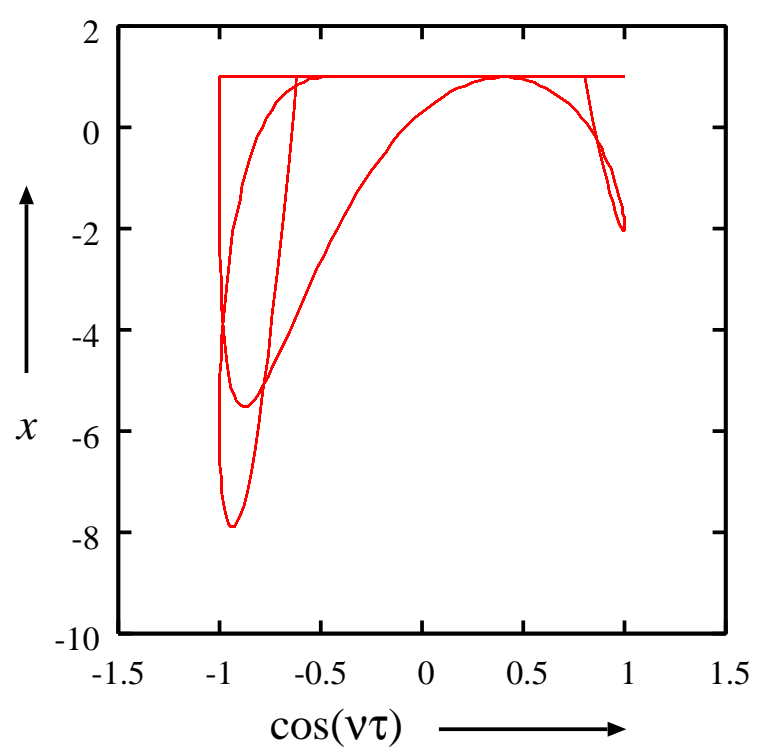

(b)

FIG. 6: Attractors obtained from Eqs. (10) and (11) with $\delta_{1}=0.1, \delta_{2}=0.5$, and $\nu=0.745$ : (a) Quasi-periodic attractor with $B=0.1$ (Maximum Lyapunov exponent is $-2.19 \times 10^{-5}$.); (b) Periodic attractor with $B=0.3$ (Maximum Lyapunov exponent is -0.181.); (c) Chaotic attractor with $B=0.38$.(Maximum Lyapunov exponent is 0.188.) 


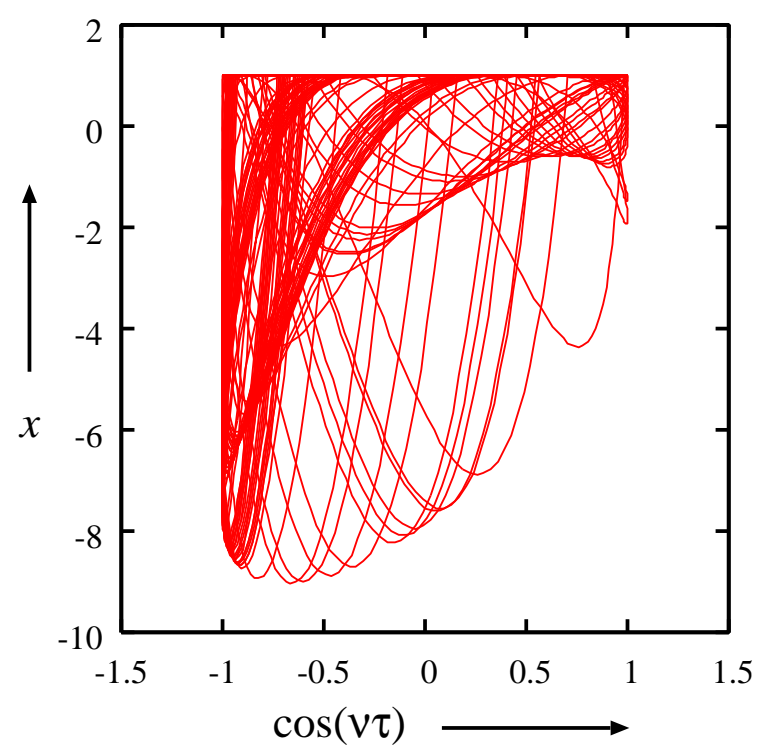

(c)

FIG. 6: - continued.

two- or higher dimensional driven oscillators because their mathematical discussions are too complex and few. On the other hand, bifurcations and chaos in the one-dimensional maps have been investigated in detail [Li \& Yorke, 1975; Myrberg, 1963; Collet \& Eckmann, 1980; Outlund et al., 1983; Kaneko, 1984; Katznelson, 1977].

Note that Eq. (10) is one-dimensional when the diode is on. Thereby, the Poincaré map is constructed as a one-dimensional map as follows. A plane, $D_{1}$, a subset, $D_{2}$, and a line, $C$, are defined as

$$
\begin{aligned}
& D_{1}=\{(\tau, x, y) \mid x=1\}, \\
& D_{2}=\{(\tau, x, y) \mid x<1\}, \\
& C=\left\{(\tau, x, y) \mid x=1, y=-2 \delta_{2}\right\} .
\end{aligned}
$$

$D_{1}$ is a plane where the diode is on, $D_{2}$ is a space where the diode is off, and line $C$ is the transition condition of Eq. (11.a), which is on $D_{1}$. Figure 7 illustrates the geometric structure of the vector field. Let us consider a flow where the initial point is on $C$. The 


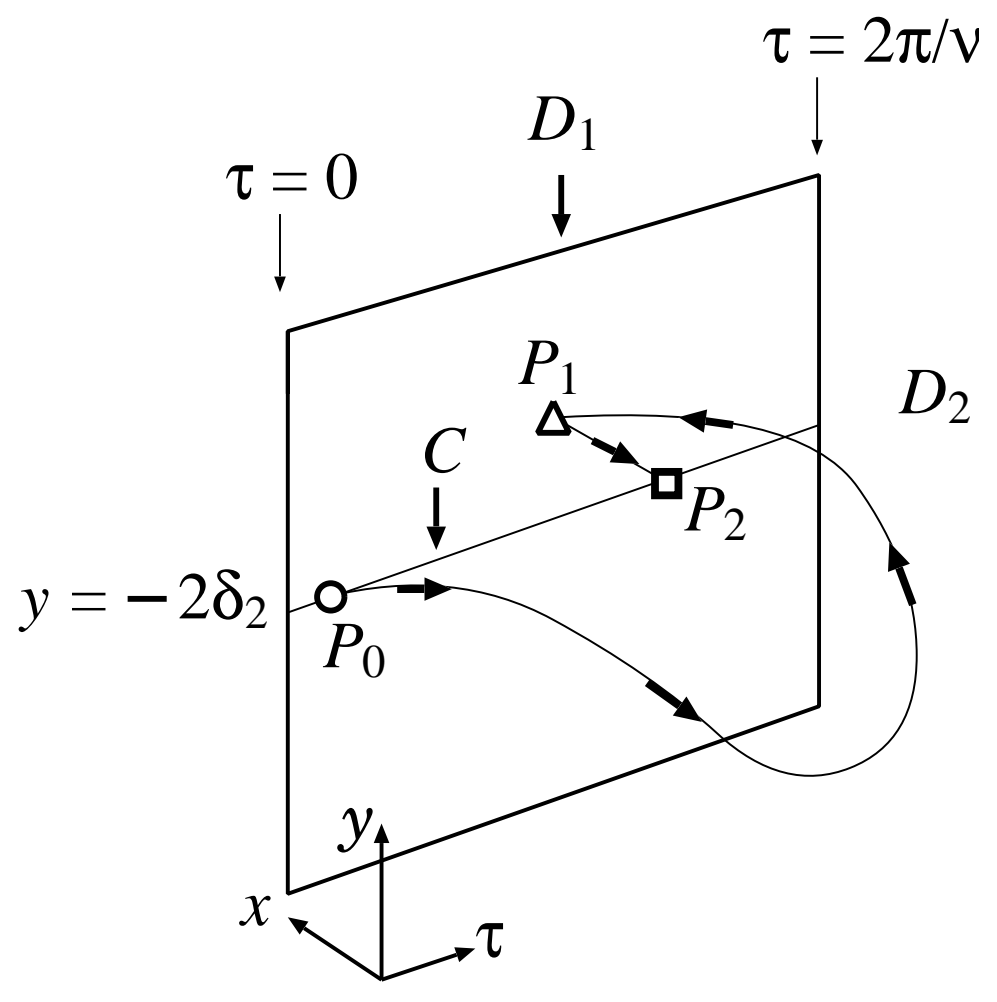

FIG. 7: Geometric structure of vector field.

initial point is denoted as $P_{0}$ in Fig. 7 . Note that $\dot{x}=0$ at any point on $C$. $\ddot{x}<0$ holds for any point on $C$ if the following condition is satisfied:

$$
4 \delta_{1} \delta_{2}-1+B<0
$$

The case where Eq. (15) is satisfied will be considered in the following discussions. In such a case, any solution which leaves $C$ enters $D_{2}$, where the diode is off $(x<1)$, and hits $D_{1}$, where the diode is on $(x=1)$, at point $P_{1}\left(\tau=\tau_{1}\right)$. At this moment, the solution is constrained on $D_{1}$, and it finally reaches $C$ again at $P_{2}\left(\tau=\tau_{2}\right)$. Therefore, the Poincaré 
map is defined by a one-dimensional map as follows:

$$
T: C \rightarrow C, \theta \mapsto T(\theta)
$$

where

$$
\begin{aligned}
\theta & =\frac{\nu}{2 \pi} \tau_{0} \text { for } \tau_{0} \in\left[0, \frac{2 \pi}{\nu}\right) \\
T(\theta) & =\frac{\nu}{2 \pi} \tau_{2} \bmod 1 .
\end{aligned}
$$

$\tau_{0}$ is the time of point $P_{0}$ and $\tau_{2}$ is the time of point $P_{2}$. Since the forcing term is periodic,

$$
T(0)=T(1)
$$

holds. Therefore, $T$ is a circle map under a certain condition which is rigorously described in Section V. We emphasize that the circle map is constructed from a simple and natural driven oscillator.

The representation of $T$ is as follows: point $P_{0}$, which is an initial point on $C$, is denoted as $(\tau, x, y)=\left(\tau_{0}, 1,-2 \delta_{2}\right)$. Let point $P_{1}$ be denoted as $(\tau, x, y)=\left(\tau_{1}, 1, y_{1}\right)$. Equations $(8)$ and (9) yield $y_{1}$ as follows:

$$
\begin{aligned}
y_{1} & =y\left(\tau_{1} ; \tau_{0}, 1,-2 \delta_{2}\right) \\
& =\left(\begin{array}{ll}
0 & 1
\end{array}\right) \times\left[\left(F_{2}\left(\tau_{1}-\tau_{0}\right) \times F_{2}(0)^{-1} \times\left\{\left(\begin{array}{c}
1 \\
-2 \delta_{2}
\end{array}\right)-G_{2}\left(\tau_{0}\right)\right\}+G_{2}\left(\tau_{1}\right)\right],\right.
\end{aligned}
$$

where $\tau_{1}$ is the minimum value that satisfies the following transcendental equation:

$$
H_{1}\left(\tau_{1}, \tau_{0}\right)=0
$$

where

$$
\begin{aligned}
& H_{1}\left(\tau_{1}, \tau_{0}\right) \equiv \\
& \left(\begin{array}{ll}
1 & 0
\end{array}\right) \times\left[F_{2}\left(\tau_{1}-\tau_{0}\right) \times F_{2}(0)^{-1} \times\left\{\left(\begin{array}{c}
1 \\
-2 \delta_{2}
\end{array}\right)-G_{2}\left(\tau_{0}\right)\right\}+G_{2}\left(\tau_{1}\right)\right]-1 .
\end{aligned}
$$


Let point $P_{2}$ be denoted as $(\tau, x, y)=\left(\tau_{2}, 1,-2 \delta_{2}\right) . \tau_{2}$ is the minimum value that satisfies the following transcendental equation:

$$
H_{2}\left(\tau_{2}, \tau_{1}, y_{1}\right)=0
$$

where

$$
\begin{aligned}
& H_{2}\left(\tau_{2}, \tau_{1}, y_{1}\right)=A e^{-2 \delta_{1}\left(\tau_{2}-\tau_{1}\right)}+k_{1 a} \sin \left(\nu \tau_{2}\right)+k_{1 b} \cos \left(\nu \tau_{2}\right)+k_{1 c}+2 \delta_{2} \\
& A=y_{1}-k_{1 a} \sin \left(\nu \tau_{1}\right)-k_{1 b} \cos \left(\nu \tau_{1}\right)-k_{1 c} .
\end{aligned}
$$

According to Eqs. (18) through (20), we can conclude that the Poincaré map is represented by the transcendental equations. It includes no approximation. Therefore, these equations can be solved to any degree using a computer.

Figure 8 illustrates the Poincaré map. The transcendental equation is solved by Newton's method. When $B$ is small, the Poincaré map is a diffeomorphism on a circle, as shown in Fig. 8 (a.1). In this case, torus is generated [Katznelson, 1977]. A quasi-periodic trajectory is drawn in Fig. 8 (a.2). On the other hand, when $B$ is large, the Poincaré map has extrema, as shown in Fig. 8 (b.1). It is well known that chaos is generated in a circle map which has extrema [Outlund et al., 1983; Kaneko, 1984]. Hence, chaos naturally occurs in this case. A chaotic trajectory of the map is drawn in Fig. 8 (b.2).

\section{BIFURCATION STRUCTURE OF DRIVEN OSCILLATOR}

In this section, the bifurcation structure of the constraint Duffing van der Pol oscillator is rigorously investigated, where the boundary between a torus-generating region and a chaosgenerating region is explicitly derived. This expression of the boundary is noteworthy for its explicitness, even though the Poincaré map is given by implicit equations of $H_{1}$ and $H_{2}$. This boundary is denoted as $\Sigma$. The definite expression of the boundary will be presented in Theorem 1, the proof of which is given in Appendix C. In the process of the proof, it 


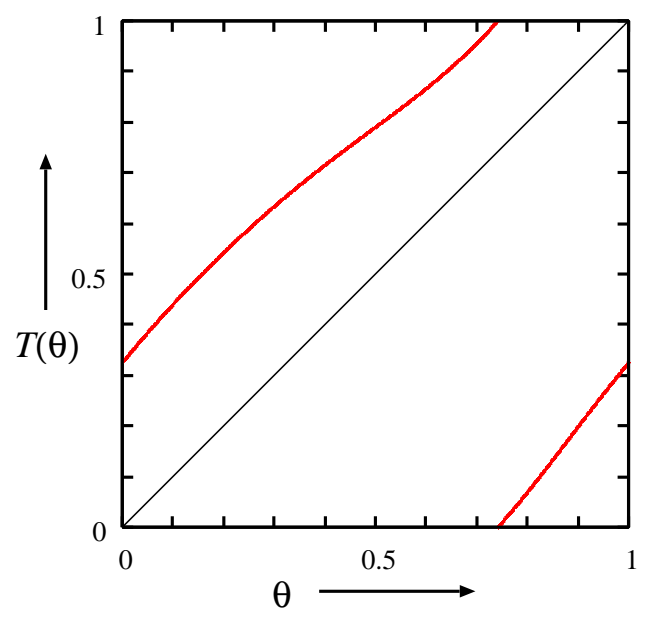

(a.1)

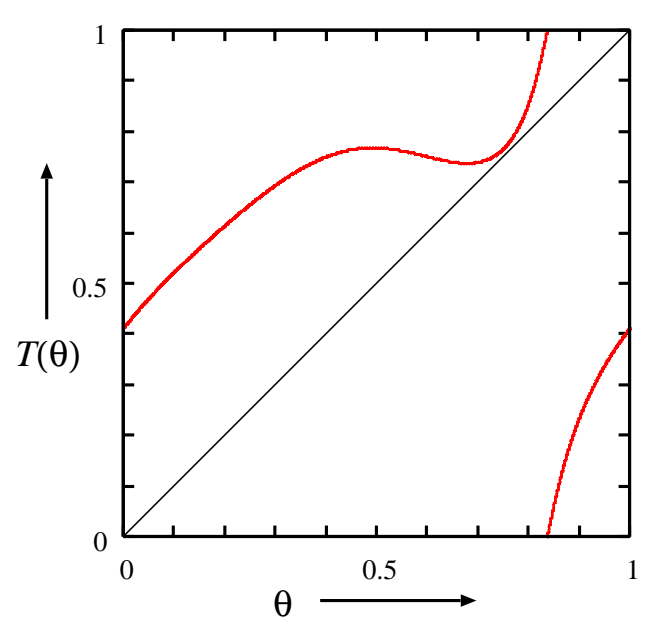

(b.1)

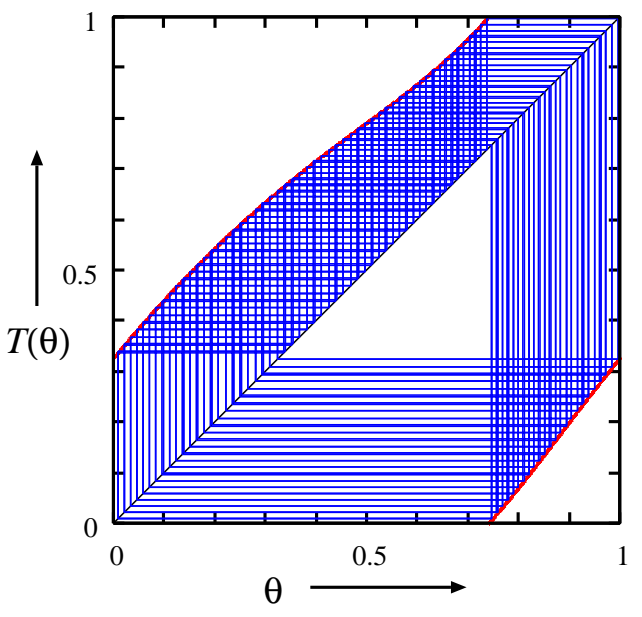

(a.2)

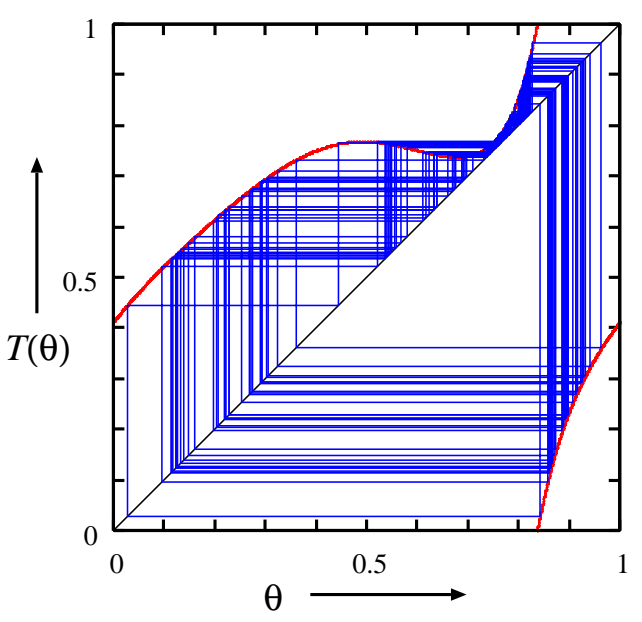

$(\mathrm{b} .2)$

FIG. 8: Poincaré maps and their trajectories with $\delta_{1}=0.1, \delta_{2}=0.5$, and $\nu=0.745$ :

(a) Diffeomorphic circle map and torus with $B=0.1\left(<B_{c}\right)$; (b) Circle map with extrema and chaos with $B=0.38\left(>B_{c}\right)$.

is shown that the expression of the differential coefficient of $T$ with respect to $\theta$, which is denoted as $D T$, is extremely simple (see Appendix B). The main point of the proof is based on the fact that we could calculate $\tau_{1}$ explicitly such that $D T=0$ owing to such a very 
simple expression of DT (see Appendix C).

In the following discussions, the parameters $\delta_{1}, \delta_{2}$ and $\nu$ are fixed and $B$ is chosen as a bifurcation parameter. To simplify the discussion, continuity of the Poincaré map is assumed. We must first derive the range of $B$ that supports the continuity of the Poincaré map.

Let us consider the solution of the linear differential equation (10.a) where the initial point is on $C$. After leaving $C, x\left(\tau ; \tau_{0}, 1,-2 \delta_{2}\right)$ becomes a minimum at $\tau=\tau_{a}$, where $\tau_{a}$ is the first solution of $\dot{x}\left(\tau_{a} ; \tau_{0}, 1,-2 \delta_{2}\right)=0$, and then it becomes a maximum at $\tau=\tau_{b}$, where $\tau_{b}$ is the second solution of $\dot{x}\left(\tau_{b} ; \tau_{0}, 1,-2 \delta_{2}\right)=0$. Let $x_{s}$ be this first maximum at $\tau=\tau_{b}$. Let the least value of $B$ that satisfies

$$
\min _{\tau_{0}} x_{s}\left(\tau_{0}\right)=1 \text { for all } \tau_{0} \text { which satisfy } \frac{d x_{s}}{d \tau_{0}}=0 \text { and } \frac{d^{2} x_{s}}{d \tau_{0}{ }^{2}}>0
$$

be denoted as $B_{\max }$.

Proposition 1 The Poincaré map $T$ is differentiable if

$$
0<B<B_{\max }
$$

See Appendix A for the proof of Proposition 1.

The most important result in this paper is the following theorem.

Theorem 1 Choose the system parameters $\delta_{1}, \delta_{2}, \nu$ and $B$ such that

$$
\begin{aligned}
& \sqrt{1-\left(\delta_{1}+\delta_{2}\right)^{2}}\left(\tau_{1}-\tau_{0}\right)+\varphi_{0}<3 \pi, \quad \forall \tau_{0} \in[0,2 \pi / \nu) \\
& \text { where } \quad \tan \varphi_{0}=-\frac{\sqrt{1-\left(\delta_{1}+\delta_{2}\right)^{2}}}{\delta_{1}+\delta_{2}}\left(\pi / 2<\varphi_{0}<\pi\right) .
\end{aligned}
$$


$\delta_{1}, \delta_{2}$, and $\nu$ are fixed and $B$ is chosen as a bifurcation parameter. Define $B_{c}$ as

$$
\begin{aligned}
B_{c}= & B_{c}\left(\delta_{1}, \delta_{2}, \nu\right) \\
= & \left(1+2 \delta_{1} e^{\frac{\left(\delta_{2}-\delta_{1}\right) \varphi_{1}}{\sqrt{1-\left(\delta_{1}+\delta_{2}\right)^{2}}}}\right) \\
& \times \sqrt{\frac{\left(1-4 \delta_{1} \delta_{2}-\nu^{2}\right)^{2}+4\left(\delta_{2}-\delta_{1}\right)^{2} \nu^{2}}{\left(4 \delta_{1}^{2}+\nu^{2}\right) e^{\frac{2\left(\delta_{2}-\delta_{1}\right) \varphi_{1}}{\sqrt{1-\left(\delta_{1}+\delta_{2}\right)^{2}}}+\left(4 \delta_{1} \cos \frac{\nu \varphi_{1}}{\sqrt{1-\left(\delta_{1}+\delta_{2}\right)^{2}}}+2 \nu \sin \frac{\nu \varphi_{1}}{\sqrt{1-\left(\delta_{1}+\delta_{2}\right)^{2}}}\right) e^{\frac{\left(\delta_{2}-\delta_{1}\right) \varphi_{1}}{\sqrt{1-\left(\delta_{1}+\delta_{2}\right)^{2}}}}+1}},}
\end{aligned}
$$

where $\varphi_{1}=2 \pi-\varphi_{0}$.

$T$ is a diffeomorphism on a circle for $0 \leq B<B_{c}$, and $T$ is a circle map which has extrema for $B_{c}<B<B_{\max }$.

The proof of the theorem is given in Appendix C. It is well known that a circle map generates torus when it is a diffeomorphism, and that it generates chaos when it has extrema [Outlund et al., 1983; Kaneko, 1984; Katznelson, 1977]. Quasi-periodic attractors and periodic attractors are alternately generated when the circle map is a diffeomorphism, and chaotic attractors and periodic attractors are alternately generated when the circle map has extrema. Therefore, $B=B_{c}\left(\delta_{1}, \delta_{2}, \nu\right)$ is the boundary between a torus-generating region and a chaos-generating region, namely, $\Sigma$.

Now, a bifurcation diagram is illustrated. The Poincaré map depends on the system parameter $\lambda$, which must explicitly appear in the map as $T(\theta, \lambda)$. The saddle-node bifurcation point for the solution of period $m$ is obtained by solving the following bifurcation equation:

$$
\left\{\begin{array}{l}
T^{m}(\theta, \lambda)-\theta=0 \\
D T^{m}(\theta, \lambda)-1=0
\end{array}\right.
$$

where $T^{m}$ is an $m$-times composite of $T$ and $D T^{m}=\frac{d T^{m}}{d \theta}$. The period-doubling bifurcation point is obtained by solving the following bifurcation equation:

$$
\left\{\begin{array}{l}
T^{m}(\theta, \lambda)-\theta=0 \\
D T^{m}(\theta, \lambda)+1=0
\end{array}\right.
$$


$D T$ is calculated and represented by the following very simple form:

$$
\begin{aligned}
D T(\theta) & =\frac{d T(\theta)}{d \theta} \\
& =\frac{4 \delta_{1} \delta_{2}-1+B \cos \left(\nu \tau_{0}\right)}{\gamma\left(4 \delta_{1} \delta_{2}-1+B \cos \left(\nu \tau_{2}\right)\right)} e^{\delta\left(\tau_{1}-\tau_{0}\right)-2 \delta_{1}\left(\tau_{2}-\tau_{1}\right)} \sin \left\{\gamma\left(\tau_{1}-\tau_{0}\right)+\varphi_{0}\right\} \\
& \text { where } \quad \tan \varphi_{0}=-\frac{\sqrt{1-\left(\delta_{1}+\delta_{2}\right)^{2}}}{\delta_{1}+\delta_{2}} \quad\left(\pi / 2<\varphi_{0}<\pi\right) .
\end{aligned}
$$

The derivation of $D T$ is presented in Appendix B. $D T^{m}(\theta)$ is calculated by the chain rule and is represented by

$$
D T^{m}(\theta)=D T(\theta) \cdot D T(T(\theta)) \cdot D T\left(T^{2}(\theta)\right) \cdot \ldots \cdot D T\left(T^{m-1}(\theta)\right)
$$

Note that bifurcation equations (25) and (26) include no approximation. We can solve bifurcation equations (25) and (26) to any degree of precision using a computer.

Figures 9 illustrates a two-parameter bifurcation diagram. In this figure, the abscissa denotes the angular frequency $\nu$ of forcing term, and the ordinate denotes its amplitude B. Many ultraharmonic, subharmonic, and fractional harmonic entrainments are observed in this diagram. These entrainments are called Arnol'd tongues. The boundary of these entrainments is generally the saddle-node bifurcations. These curves are denoted by " $G$ " in the figure. Furthermore, "I" denotes the period-doubling bifurcation curve. It is well known that each Arnol'd tongue is surrounded by two G-curves originating from the rational point for $B=0$ at which $\nu / \nu_{0}$ is a rational number where $\nu_{0}$ is the natural oscillation frequency of the autonomous system. In the figure, the entrainment denoted as $1 / 1$ is the fundamental harmonic entrainment, and those denoted as $2 / 1$ and $3 / 1$ are the second-harmonic and the third-harmonic entrainment, respectively. Furthermore, the entrainments denoted as 3/2, $3 / 4,2 / 3$ and $3 / 5$ are the fractional harmonic entrainments. $B_{\max }$ and $B_{c}$ are also shown in 


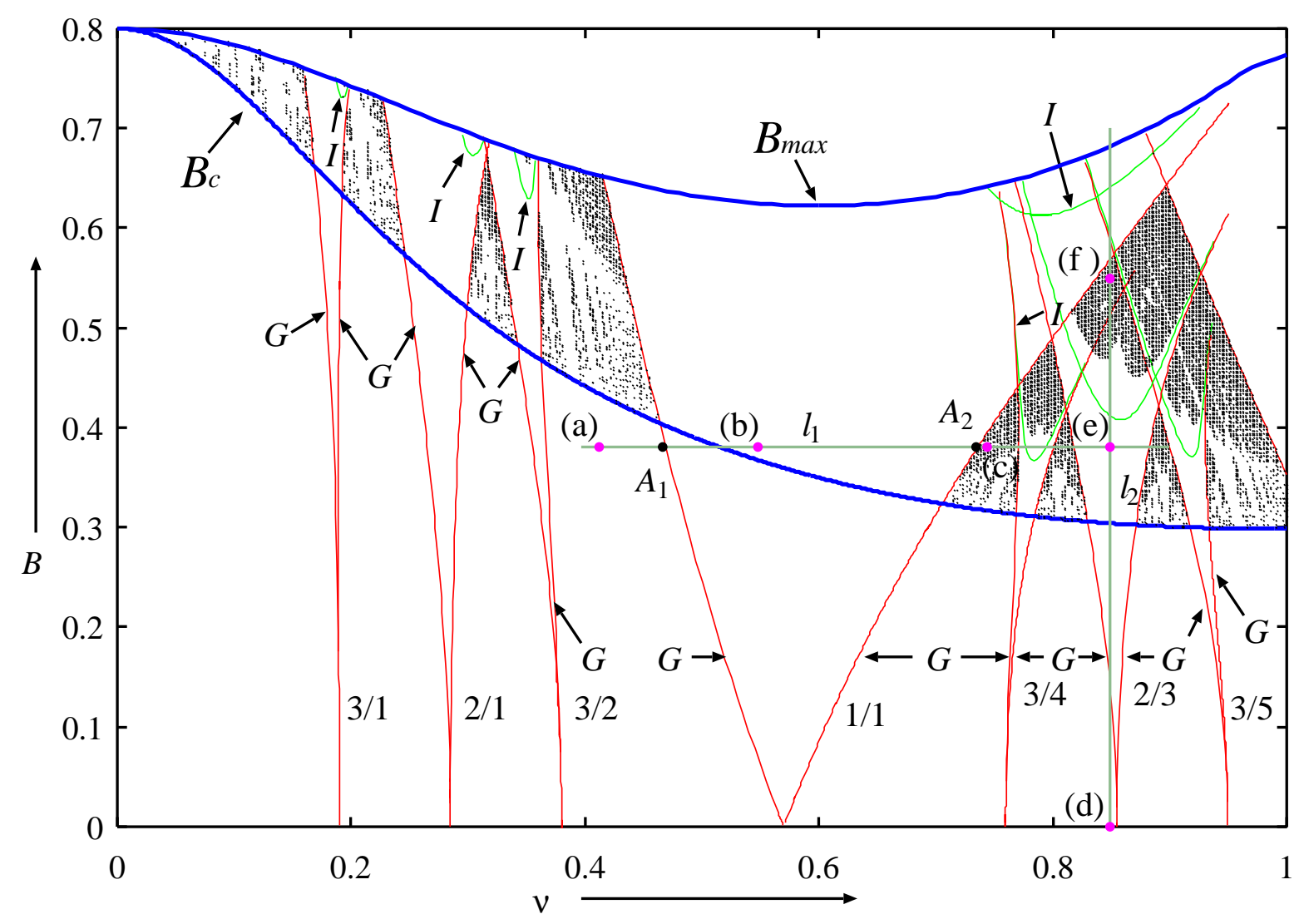

FIG. 9: Two-parameter $\nu-B$ bifurcation diagram with $\delta_{1}=0.1$ and $\delta_{2}=0.5$.

Fig. 9. The maximum Lyapunov exponent $\tilde{\lambda}$ is calculated by

$$
\begin{aligned}
\tilde{\lambda} & =\lim _{N \rightarrow \infty} \frac{1}{N} \sum_{k=1}^{N} \log \left|D T\left(\theta_{k}\right)\right| \\
& \simeq \frac{1}{10000} \sum_{k=1000}^{11000} \log \left|D T\left(\theta_{k}\right)\right|,
\end{aligned}
$$

where $\theta_{k}=T^{k}\left(\theta_{0}\right)$,

and the chaos-generating region $(\tilde{\lambda}>0)$ is shaded in Fig. 9. Chaos is observed where $B_{c}<B<B_{\max }$. This result agrees with Theorem 1. Examples of the attractors and the Poincaré maps observed at points (a), (b), ..., (f) in the figure are shown in Fig. 10.

Figure 11 illustrate a one-parameter bifurcation diagram of $\theta_{k}(k=1001,1002, \ldots, 1010)$ to $\nu$ and a behavior of the maximum Lyapunov exponent $\tilde{\lambda}$, where $B$ is fixed at 0.38 . This 

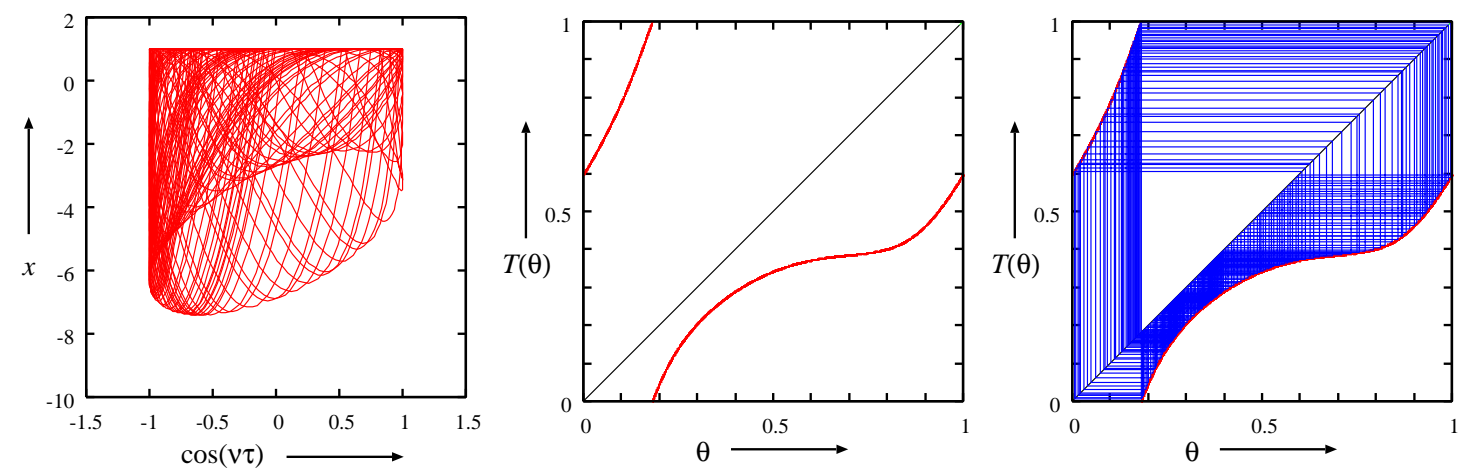

(a)
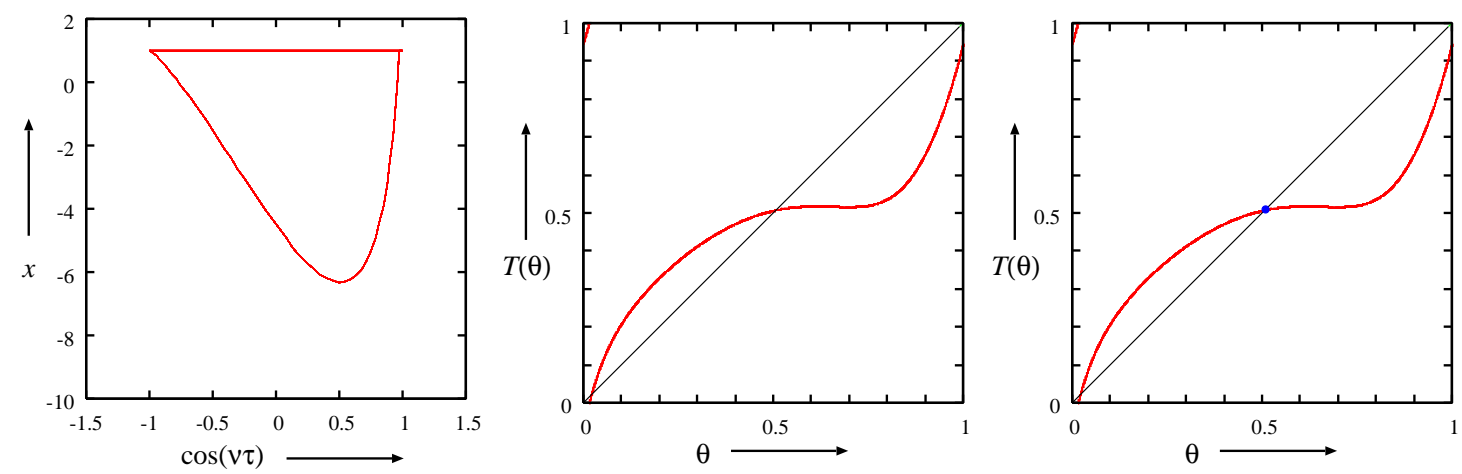

(b)
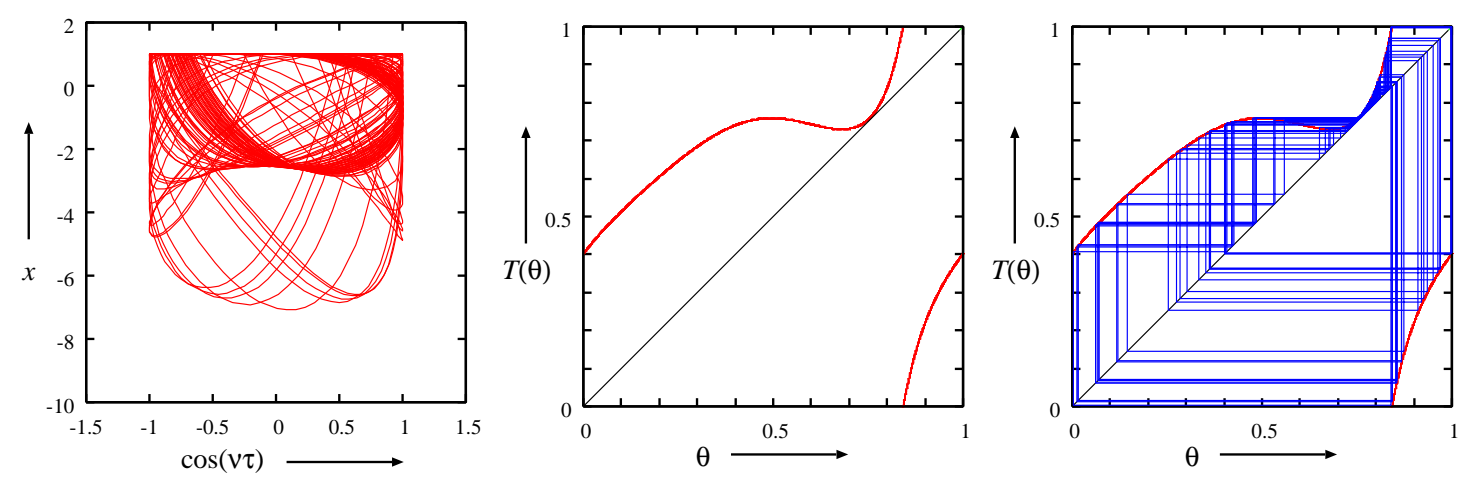

(c)

FIG. 10: Examples of attractors and Poincaré maps with $\delta_{1}=0.1, \delta_{2}=0.5$ :

(a) Quasi-periodic attractor, Poincaré map and its trajectory with $\nu=0.415$ and $B=0.38$;

(b) Periodic attractor, Poincaré map and its trajectory with $\nu=0.55$ and $B=0.38$;

(c) Chaotic attractor, Poincaré map and its trajectory with $\nu=0.74$ and $B=0.38$;

(d) Quasi-periodic attractor, Poincaré map and its trajectory with $\nu=0.85$ and $B=0.0$; 26

(e) Periodic attractor, Poincaré map and its trajectory with $\nu=0.85$ and $B=0.38$;

(f) Chaotic attractor, Poincaré map and its trajectory with $\nu=0.85$ and $B=0.55$. 



(d)
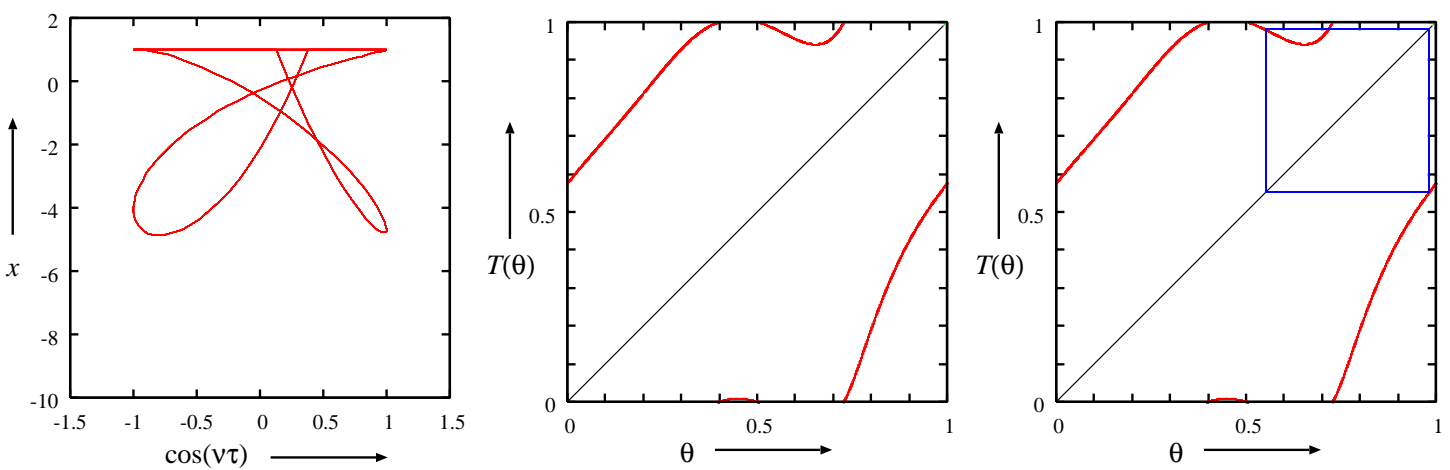

(e)
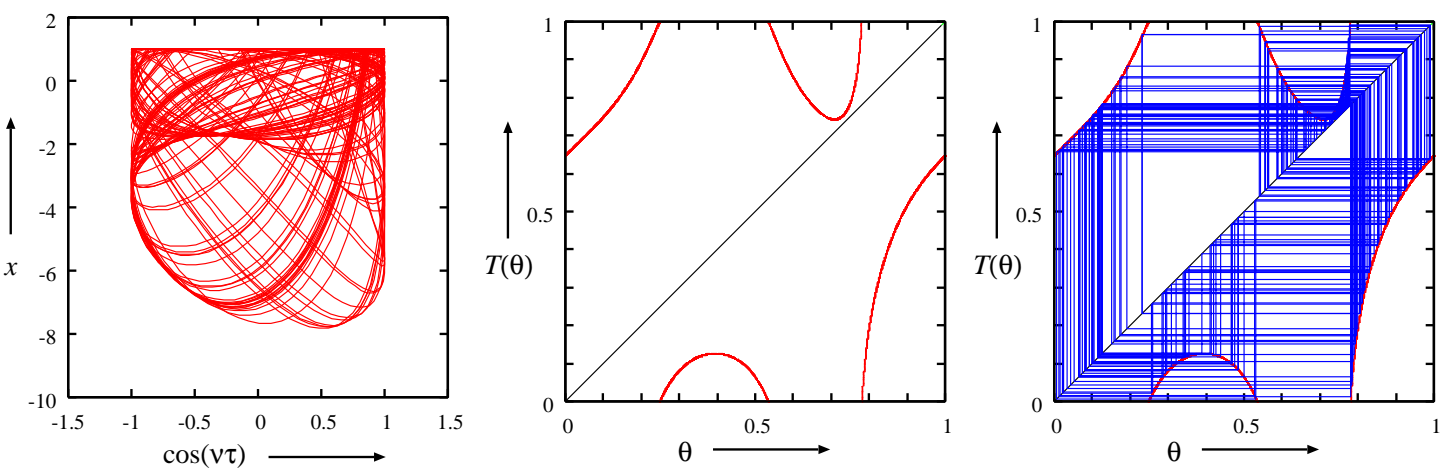

(f)

FIG. 10: - continued.

condition is indicated by line $l_{1}$ in Fig. 9. The values of $\nu_{1}$ and $\nu_{2}$ indicated in Fig. 11 correspond to points $A_{1}, A_{2}$ indicated in Fig. 9, respectively.

Nonperiodic oscillation is observed for $\nu<\nu_{1}$ and $\nu>\nu_{2}$. According to Theorem 1, we 
can draw the following conclusions. The nonperiodic oscillation for $\nu<\nu_{1}$ is quasi-periodic, because point $A_{1}$ in Fig. 9, which corresponds to $\nu_{1}$ in Fig. 11, is located under $B_{c}$. On the other hand, the nonperiodic oscillation for $\nu>\nu_{2}$ is chaotic, because point $A_{2}$ in Fig. 9, which corresponds to $\nu_{2}$ in Fig. 11 is located over $B_{c}$. Therefore, the bifurcation at point $A_{1}$ is a saddle-node bifurcation from a periodic solution to a quasi-periodic solution, and the bifurcation at point $A_{2}$ is an intermittency. Figures 12 (a) and (b) illustrate the Poincaré maps obtained at points $A_{1}$ and $A_{2}$ in Fig. 9, respectively.

Figure 13 illustrates a one-parameter bifurcation diagram of $\theta_{k}(k=1001,1002, \ldots$, 1010) to $B$ and a behavior of the maximum Lyapunov exponent $\tilde{\lambda}$, where $\nu$ is fixed at 0.85 . This condition is indicated by line $l_{2}$ in Fig. 9. The period-doubling-bifurcations route to chaos is observed.

\section{APPENDIX A: DIFFERENTIABILITY OF $T$}

\section{PROOF OF PROPOSITION 1.}

If both Eqs. (A1) and (A2) are satisfied for any $\tau_{0}$,

$$
\begin{aligned}
& \frac{\partial H_{1}}{\partial \tau_{1}} \neq 0, \\
& \frac{\partial H_{2}}{\partial \tau_{2}} \neq 0,
\end{aligned}
$$

then, by the implicit function theorem, the Poincaré map $T(\theta)$ is differentiable on $[0,1)$ since all the functions of Eqs. (16), (18), (19), and (20), which give the relations between $\theta$ and $T(\theta)$, are totally differentiable. It is easily checked that Eq. (A2) is always satisfied from Eq. (15). On the other hand, $\partial H_{1} / \partial \tau_{1}$ is calculated as

$$
\frac{\partial H_{1}}{\partial \tau_{1}}=2 \delta_{2}+y_{1} .
$$




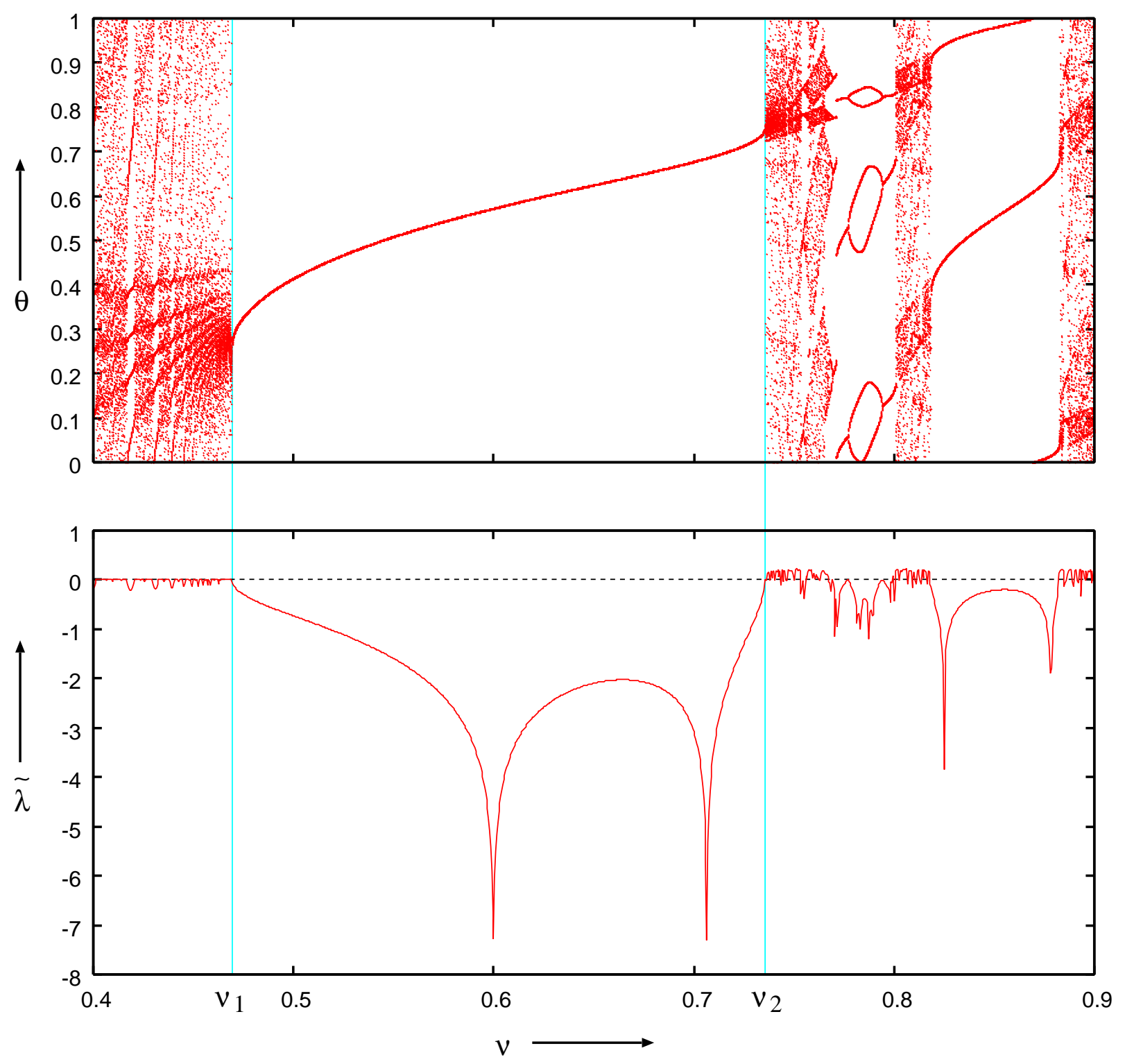

FIG. 11: One-parameter bifurcation diagram and maximum Lyapunov exponent with

$$
\delta_{1}=0.1, \delta_{2}=0.5 \text { and } B=0.38 \text {. }
$$

Now, for $0 \leq B<B_{\text {max }}, \exists \tau_{s}>\tau_{1}$ holds from $x\left(\tau_{1}\right)=1$ and $x_{s}>1$. Since $\dot{x}(\tau)>0$ for $\tau_{1} \leq \tau<\tau_{s}$, we get $\dot{x}\left(\tau_{1}\right)=2 \delta_{2}+y_{1}>0$. Therefore Eq. (A1) holds. 




(a)

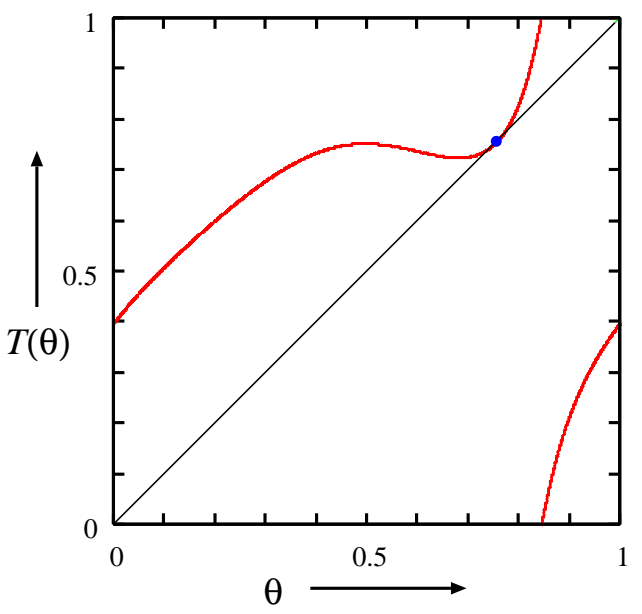

(b)

FIG. 12: Poincaré maps with $\delta_{1}=0.1, \delta_{2}=0.5$, and $B=0.38$ : (a) Poincaré map at point $A_{1}$ in Fig. 9 with $\nu=\nu_{1} \simeq 0.469137$; (b) Poincaré map at point $A_{2}$ in Fig. 9 with

$$
\nu=\nu_{2} \simeq 0.735481
$$

\section{APPENDIX B: DERIVATION OF $D T$}

Equation (27) is derived as follows:

$$
\begin{aligned}
D T(\theta) & =\frac{d T(\theta)}{d \theta} \\
& =\frac{\nu}{2 \pi} \frac{d \tau_{2}}{d \tau_{0}} \frac{2 \pi}{\nu} \\
& =\frac{d \tau_{2}}{d \tau_{0}} \\
& =\frac{\partial \tau_{2}\left(\tau_{1}, y_{1}\right)}{\partial \tau_{1}} \frac{d \tau_{1}}{d \tau_{0}}+\frac{\partial \tau_{2}\left(\tau_{1}, y_{1}\right)}{\partial y_{1}} \frac{d y_{1}}{d \tau_{0}} \\
& =\frac{\partial \tau_{2}\left(\tau_{1}, y_{1}\right)}{\partial \tau_{1}} \frac{d \tau_{1}}{d \tau_{0}}+\frac{\partial \tau_{2}\left(\tau_{1}, y_{1}\right)}{\partial y_{1}}\left(\frac{\partial y_{1}\left(\tau_{0}, \tau_{1}\right)}{\partial \tau_{0}}+\frac{\partial y_{1}\left(\tau_{0}, \tau_{1}\right)}{\partial \tau_{1}} \frac{d \tau_{1}}{d \tau_{0}}\right)
\end{aligned}
$$

where

$$
\frac{\partial \tau_{2}}{\partial \tau_{1}}=-\frac{\partial H_{2} / \partial \tau_{1}}{\partial H_{2} / \partial \tau_{2}}, \quad \frac{\partial \tau_{2}}{\partial y_{1}}=-\frac{\partial H_{2} / \partial y_{1}}{\partial H_{2} / \partial \tau_{2}} \quad \text { and } \quad \frac{\partial \tau_{1}}{\partial \tau_{0}}=-\frac{\partial H_{1} / \partial \tau_{0}}{\partial H_{1} / \partial \tau_{1}}
$$





FIG. 13: One-parameter bifurcation diagram and maximum Lyapunov exponent with

$$
\delta_{1}=0.1, \delta_{2}=0.5 \text { and } \nu=0.85 .
$$

By simple calculation, we find

$$
\begin{aligned}
\frac{\partial \tau_{2}\left(\tau_{1}, y_{1}\right)}{\partial \tau_{1}} & =-\frac{\partial \tau_{2}\left(\tau_{1}, y_{1}\right)}{\partial y_{1}} \frac{\partial y_{1}\left(\tau_{0}, \tau_{1}\right)}{\partial \tau_{1}}, \\
-\frac{\partial H_{2} / \partial y_{1}}{\partial H_{2} / \partial \tau_{2}} & =-\frac{e^{-2 \delta_{1}\left(\tau_{2}-\tau_{1}\right)}}{4 \delta_{1} \delta_{2}-1+B \cos \left(\nu \tau_{2}\right)} .
\end{aligned}
$$


Substituting Eq. (B3) into Eqs. (B1) and (B2) yields

$$
\begin{aligned}
D T(\theta)= & \frac{\partial \tau_{2}\left(\tau_{1}, y_{1}\right)}{\partial y_{1}} \frac{\partial y_{1}\left(\tau_{0}, \tau_{1}\right)}{\partial \tau_{0}} \\
= & -\frac{\partial H_{2} / \partial y_{1}}{\partial H_{2} / \partial \tau_{2}} \frac{\partial y_{1}\left(\tau_{0}, \tau_{1}\right)}{\partial \tau_{0}} \\
= & \frac{4 \delta_{1} \delta_{2}-1+B \cos \left(\nu \tau_{0}\right)}{\gamma\left(4 \delta_{1} \delta_{2}-1+B \cos \left(\nu \tau_{2}\right)\right)} e^{\delta\left(\tau_{1}-\tau_{0}\right)-2 \delta_{1}\left(\tau_{2}-\tau_{1}\right)} \sin \left\{\gamma\left(\tau_{1}-\tau_{0}\right)+\varphi_{0}\right\} \\
& \text { where } \quad \tan \varphi_{0}=-\frac{\sqrt{1-\left(\delta_{1}+\delta_{2}\right)^{2}}}{\delta_{1}+\delta_{2}} \quad\left(\pi / 2<\varphi_{0}<\pi\right) .
\end{aligned}
$$

\section{APPENDIX C: PROOF OF THEOREM 1}

\section{PROOF.}

A naive way of obtaining boundary $\Sigma$ is to solve

$$
D T(\theta, \lambda)=0 \text { and } D^{2} T(\theta, \lambda)=0
$$

where $D^{2} T=\frac{d}{d \theta} D T . D T(\theta, \lambda)$ and $D^{2} T(\theta, \lambda)$ may be derived as implicit equations. Therefore, $\Sigma$ may be obtained by solving these equations numerically, not explicitly. On the other hand, the simple expression (B4) is utilized to obtain $\Sigma$ explicitly in the following proof [Inaba \& Mori, 1991].

Under condition (15), the coefficient of $\sin \left\{\gamma\left(\tau_{1}-\tau_{0}\right)+\varphi_{0}\right\}$ in Eq. (B4), such as

$$
\frac{4 \delta_{1} \delta_{2}-1+B \cos \left(\nu \tau_{0}\right)}{\gamma\left(4 \delta_{1} \delta_{2}-1+B \cos \left(\nu \tau_{2}\right)\right)} e^{\delta\left(\tau_{1}-\tau_{0}\right)-2 \delta_{1}\left(\tau_{2}-\tau_{1}\right)}
$$

is positive. Hence,

$$
\begin{aligned}
& D T(\theta)>0 \quad \text { if } \quad 2 \pi<\gamma\left(\tau_{1}-\tau_{0}\right)+\varphi_{0}<3 \pi \\
& D T(\theta)<0 \quad \text { if } \quad \pi<\gamma\left(\tau_{1}-\tau_{0}\right)+\varphi_{0}<2 \pi,
\end{aligned}
$$

according to the sign of $\sin \left\{\gamma\left(\tau_{1}-\tau_{0}\right)+\varphi_{0}\right\}$. Therefore, if

$$
2 \pi<\gamma\left(\tau_{1}-\tau_{0}\right)+\varphi_{0}<3 \pi \quad \forall \tau_{0}
$$


$T$ is a diffeomorphism, and if

$$
\pi<\gamma\left(\tau_{1}-\tau_{0}\right)+\varphi_{0}<2 \pi \quad \exists \tau_{0}
$$

$T$ has extrema. The following statement holds.

- $T$ is a diffeomorphism, if

$$
H_{1}\left(\tilde{\tau}_{1}\left(\tau_{0}\right), \tau_{0}\right) \neq 0 \forall \tau_{0} \in[0,2 \pi / \nu)
$$

- $T$ has extrema, if

$$
H_{1}\left(\tilde{\tau}_{1}\left(\tau_{0}\right), \tau_{0}\right)=0 \exists \tau_{0} \in[0,2 \pi / \nu)
$$

where

$$
\gamma\left(\tilde{\tau}_{1}-\tau_{0}\right)+\varphi_{0}=2 \pi
$$

namely

$$
\tilde{\tau}_{1}\left(\tau_{0}\right)=\tau_{0}+\frac{2 \pi-\varphi_{0}}{\gamma}
$$

Note that $\tilde{\tau}_{1}\left(\tau_{0}\right)$ is $\tau_{1}$ such that $D T(\theta, \lambda)=0$. Thus, we can avoid solving the implicit equations.

We will calculate $H_{1}\left(\tilde{\tau}_{1}\left(\tau_{0}\right), \tau_{0}\right)$. From easy calculations, the following relations hold:

$$
\begin{aligned}
& \sin \left\{\gamma\left(\tilde{\tau}_{1}-\tau_{0}\right)\right\}=-\gamma, \\
& \cos \left\{\gamma\left(\tilde{\tau}_{1}-\tau_{0}\right)\right\}=-\left(\delta_{1}+\delta_{2}\right), \\
& \sin \left(\nu \tilde{\tau}_{1}\right)=\cos \frac{\nu \varphi_{1}}{\gamma} \sin \left(\nu \tau_{0}\right)+\sin \frac{\nu \varphi_{1}}{\gamma} \cos \left(\nu \tau_{0}\right), \\
& \cos \left(\nu \tilde{\tau}_{1}\right)=\cos \frac{\nu \varphi_{1}}{\gamma} \cos \left(\omega \tau_{0}\right)-\sin \frac{\nu \varphi_{1}}{\gamma} \sin \left(\nu \tau_{0}\right), \\
& \text { where } \varphi_{1}=2 \pi-\varphi_{0} .
\end{aligned}
$$


Substituting Eq. (C8) into $H_{1}\left(\tilde{\tau}_{1}\left(\tau_{0}\right), \tau_{0}\right)$ and calculating it, we obtain

$$
\begin{aligned}
H_{1}\left(\tilde{\tau}_{1}, \tau_{0}\right)= & -2 \delta_{1} e^{\frac{\delta \varphi_{1}}{\gamma}}+\left\{\left(2 \delta_{1} k_{2 a}-\nu k_{2 b}\right) e^{\frac{\nu \varphi_{1}}{\gamma}}+k_{2 a} \cos \frac{\nu \varphi_{1}}{\gamma}-k_{2 b} \sin \frac{\nu \varphi_{1}}{\gamma}\right\} \sin \left(\nu \tau_{0}\right) \\
& +\left\{\left(2 \delta_{1} k_{2 a}-\nu k_{2 b}\right) e^{\frac{\delta \varphi_{1}}{\gamma}}+k_{2 a} \sin \frac{\nu \varphi_{1}}{\gamma}+k_{2 b} \cos \frac{\nu \varphi_{1}}{\gamma}\right\} \cos \left(\nu \tau_{0}\right)-1 \\
= & A\left(B, \delta_{1}, \delta_{2}, \nu\right) \sin \left(\nu \tau_{0}+\phi\right)-1-2 \delta_{1} e^{\frac{\left(\delta_{2}-\delta_{1}\right) \varphi_{1}}{\sqrt{1-\left(\delta_{1}+\delta_{2}\right)^{2}}}},
\end{aligned}
$$

where

$$
\begin{aligned}
& A\left(B, \delta_{1}, \delta_{2}, \nu\right)=\frac{B}{\sqrt{\left(1-4 \delta_{1} \delta_{2}-\nu^{2}\right)^{2}+4\left(\delta_{2}-\delta_{1}\right)^{2} \nu^{2}}} \\
& \times \sqrt{\left(4 \delta_{1}^{2}+\nu^{2}\right) e^{\frac{2\left(\delta_{2}-\delta_{1}\right) \varphi_{1}}{\sqrt{1-\left(\delta_{1}+\delta_{2}\right)^{2}}}+\left(4 \delta_{1} \cos \frac{\nu \varphi_{1}}{\sqrt{1-\left(\delta_{1}+\delta_{2}\right)^{2}}}+2 \nu \sin \frac{\nu \varphi_{1}}{\sqrt{1-\left(\delta_{1}+\delta_{2}\right)^{2}}}\right) e^{\frac{\left(\delta_{2}-\delta_{1}\right) \varphi_{1}}{\sqrt{1-\left(\delta_{1}+\delta_{2}\right)^{2}}}}+1}} \\
& \tan \phi=\frac{\left(2 \delta_{1} k_{2 a}+\nu k_{2 b}\right) e^{\frac{\delta \varphi_{1}}{\gamma}}+k_{2 a} \sin \frac{\nu \varphi_{1}}{\gamma}+k_{2 b} \cos \frac{\nu \varphi_{1}}{\gamma}}{\left(2 \delta_{1} k_{2 a}-\nu k_{2 b}\right) e^{\frac{\delta \varphi_{1}}{\gamma}}+k_{2 a} \cos \frac{\nu \varphi_{1}}{\gamma}-k_{2 b} \sin \frac{\nu \varphi_{1}}{\gamma}}
\end{aligned}
$$

In Eq. (C9), the range of $\sin \left(\nu \tau_{0}+\phi\right)$ is $[-1,1]$. Therefore, if

$$
0<A\left(B, \delta_{1}, \delta_{2}, \nu\right)<1+2 \delta_{1} e^{\frac{\left(\delta_{2}-\delta_{1}\right) \varphi_{1}}{\sqrt{1-\left(\delta_{1}+\delta_{2}\right)^{2}}}}
$$

$H_{1}\left(\tilde{\tau}_{1}\left(\tau_{0}\right), \tau_{0}\right) \neq 0 \forall \tau_{0} \in[0,2 \pi / \nu)$ and $T(\theta)$ is a diffeomorphism, and if

$$
A\left(B, \delta_{1}, \delta_{2}, \nu\right)>1+2 \delta_{1} e^{\frac{\left(\delta_{2}-\delta_{1}\right) \varphi_{1}}{\sqrt{1-\left(\delta_{1}+\delta_{2}\right)^{2}}}}
$$

$H_{1}\left(\tilde{\tau}_{1}\left(\tau_{0}\right), \tau_{0}\right)=0$ for two $\tau_{0}$ 's $\in[0,2 \pi / \nu)$ and $T(\theta)$ has extrema. By solving

$$
A\left(B_{c}, \delta_{1}, \delta_{2}, \nu\right)=1+2 \delta_{1} e^{\frac{\left(\delta_{2}-\delta_{1}\right) \varphi_{1}}{\sqrt{1-\left(\delta_{1}+\delta_{2}\right)^{2}}}}
$$

the critical parameter $B_{c}(\Sigma)$ is obtained.

\section{REFERENCE}

Alsedà, L. \& Falcó, A. [1994] "Devil staircase route to chaos in a forced relaxation oscillator," Annales de l'institut Fourier 44, 109-128. 
Collet, P. \& Eckmann, J. P. [1980] Iterated maps on the interval as dynamical systems (Birkhäuser Boston, Boston), Progress in Physics, vol. 1.

Endo, T. \& Chua, L. O. [1988] "Chaos from phase locked loop," IEEE Trans. Circuits Syst. CAS-35, 987-1003.

Inaba, N. \& Mori, S. [1988] "Chaotic phenomena in a circuit with a diode due to the change of the oscillation frequency," Trans. Inst. Electron. Inform. Communi. Engnr. Jpn. E71, 842-849.

Inaba, N., Saito, T., \& Mori, S. [1987] "Chaotic phenomena in a circuit with a linear negative resistance and an ideal switch of diodes," Trans. Inst. Electron. Inform. Communi. Engnr. Jpn. E70, 744-754.

Inaba, N. \& Mori, S. [1991] "Chaos via torus breakdown in a piecewise-linear forced van der Pol oscillator with a diode," IEEE Trans. Circuits Syst. 38, 398-409.

Kaneko, K. [1984] "Supercritical behavior of disordered orbits of a circle map," Prog. Theor. Phys. 72, 1089-1103.

Katznelson, Y. [1977] "Sigma-finite invariant measures from smooth mapping of the circle," J. D'Analyse Math. 31, 1-8.

Kawakami, H. [1984] "Bifurcation of periodic responses in forced dynamic nonlinear circuits: computation of bifurcation values of the system parameters," IEEE Trans. Circuits Syst. CAS-31, 248-260.

Levi, M. [1981] "Qualitative analysis of the periodically forced relaxation oscillations," Mem. Amer. Math. Soc. 214, 1-47.

Li, T. Y., \& Yorke, J. A. [1975], "Period three implies chaos," Amer. Math. Monthly 82, 985-992. 
Mira, C., \& Qriouet, M. [1993] "On a "crossroad area-spring area" transition occurring in a Duffing-Rayleigh equation with a periodical excitation," Int. J. Bifurcation and Chaos 3, 1029-1037.

Myrberg, P.J. [1963] "Iteration von quadratwurzeloperationen. III," Ann. Acad. Sci. Fenn. Ser. A 336, 1-10.

Outlund, S., Rand, D., Sethana, J., \& Siggia, E. [1983] "Universal properties of transition from quasi-periodicity to chaos in dissipative systems," Physica 8D, 303-342.

Qriouet, M., \& Mira, C. [1994] "Fractional harmonic synchronization in the DuffingRayleigh differential equation," Int. J. Bifurcation and Chaos 4, 411-426.

Qriouet, M., \& Mira, C. [2000] "Reducible fractional harmonics generated by the nonautonomous Duffing-Raleigh equation. Pockets of reducible harmonics and Arnold's tongues," Int. J. Bifurcation and Chaos 10, 1345-1366.

Rössler, O.E. [1976] "Different types of chaos in two simple differential equations," Naturforsch, Z. 31a, 1664-1670.

Rössler, O.E. [1979] Continuous chaos-four prototype equations, in Bifurcation Theory and Applications in Scientific Disciplines, edited by Gurel, O. and Rössler. O.E. (New York Acad. Sci., New York), Ann. New York Acad. Sci., 316, pp. 376-392.

Sekikawa, M., Inaba N., \& Tsubouchi, T. [2004] "Chaos via duck solution breakdown in a piecewise linear van der Pol oscillator driven by an extremely small periodic perturbation," Physica D 194, 227-249.

Ueda, Y., \& Akamatsu, N. [1981] "Chaotically transitional phenomena in the forced negative resistance oscillator," IEEE Trans. Circuits. Syst. CAS-28, 217-224. 
Young L.-S. [1983] 'Entropy, Lyapunov exponents, and Hausdorff dimension in differentiable dynamical systems," IEEE Trans. Circuits. Syst. CAS-30, 599-607. 